\title{
COMP2CAT: hunting compact double radio sources in the local Universe ${ }^{\star}$
}

\author{
A. Jimenez-Gallardo ${ }^{1,2}$, F. Massaro ${ }^{1,2,3,4}$, A. Capetti ${ }^{2}$, M. A. Prieto ${ }^{5,6}$, A. Paggi ${ }^{1,2,3}$, R. D. Baldi ${ }^{7}$, R. Grossova ${ }^{1,8}$,
} L. Ostorero ${ }^{1,3}$, A. Siemiginowska ${ }^{9}$, and S. Viada ${ }^{1}$

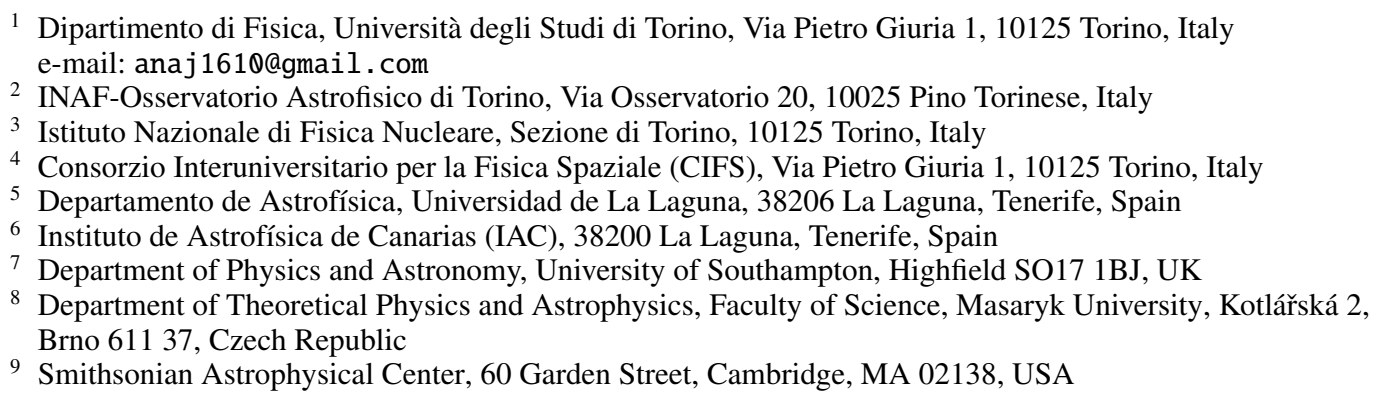

Received 22 January 2019 / Accepted 13 March 2019

\begin{abstract}
We present a catalog of compact double radio galaxies (hereafter COMP2CAT) listing 43 edge-brightened radio sources whose projected linear size does not exceed $60 \mathrm{kpc}$, the typical size of their host galaxies. This is the fifth in a series of radio source catalogs recently created, namely: FRICAT, FRIICAT, FROCAT, and WATCAT, each of which focuses on a different class of radio galaxies. The main aim of our analysis is to attain a better understanding of sources with intermediate morphologies between FR IIs and FR 0s. COMP2CAT sources were selected from an existing catalog of radio sources based on NVSS, FIRST and SDSS observations because they have (i) edge-brightened morphologies typical of FR IIs, (ii) redshifts $z<0.15$, and (iii) projected linear sizes smaller than $60 \mathrm{kpc}$. With radio luminosities at $1.4 \mathrm{GHz} 10^{38} \lesssim L_{1.4} \lesssim 10^{41} \mathrm{erg} \mathrm{s}^{-1}$, COMP2CAT sources appear as the low radio luminosity tail of FR IIs. However, their host galaxies are indistinguishable from those of large-scale radio sources: they are luminous $\left(-21 \gtrsim M_{r} \gtrsim-24\right)$, red, early-type galaxies with black hole masses in the range $10^{7.5} \lesssim M_{\mathrm{BH}} \lesssim 10^{9.5} \mathrm{M}_{\odot}$. Moreover, all but one of the COMP2CAT sources are optically classifiable as low-excitation radio galaxies, in agreement with being the low radio luminosity tail of FR Is and FR IIs. This catalog of compact double sources, which is $\sim 47 \%$ complete at $z<0.15$, can potentially be used to clarify the role of compact double sources in the general evolutionary scheme of radio galaxies.
\end{abstract}

Key words. catalogs - galaxies: active - galaxies: jets - galaxies: general

\section{Introduction}

In 1974 Fanaroff \& Riley proposed classifying extragalactic radio sources on the basis of the morphology of their extended structures at $178 \mathrm{MHz}$. These authors distinguished two main classes of radio sources: edge-darkened, known as FR Is, and edge-brightened, known as FR IIs. Fanaroff \& Riley (1974) also discovered a link between this morphological classification and the total radio luminosity, where FR Is tend to be less luminous at $178 \mathrm{MHz}$ than FR IIs. Afterward, Ledlow \& Owen (1996) found that this classification was even sharper when comparing the optical luminosity of their host galaxies with their total radio luminosity. However, several authors such as Best (2009), Lin et al. (2010), Wing \& Blanton (2011), and Capetti et al. (2017a) showed that the dichotomy in the optical-radio diagram disappears when considering samples selected at lower radio

\footnotetext{
* Table C.1 is also available at the CDS via anonymous ftp to cdsarc.u-strasbg.fr (130.79.128.5) or via http://cdsarc. u-strasbg.fr/viz-bin/qcat?]/A+A/627/A108
}

luminosity, which means that this distinction is probably due to high flux thresholds adopted in previous sample selections.

Another population, represented by "compact" radio galaxies, confined within a region of a few $\mathrm{kpc}$ and lacking large-scale jets, the formation and propagation of which is determined by plasma instabilities (see, e.g., Bodo et al. 2013, for a theoretical analysis), was later identified by Baldi et al. (2015). These sources, known as FR 0s, share almost all the characteristics of FR Is, but are lacking extended radio emission (see also Ghisellini 2011).

Motivated by the necessity of having homogeneous and complete samples of radio galaxies to investigate their properties and those of their large-scale environments, we recently compiled several different catalogs for FR Is (Capetti et al. 2017b), FR IIs (Capetti et al. 2017a), and FR 0s (Baldi et al. 2018).

The FRICAT lists 219 sources, all hosted in red early-type galaxies, spectroscopically classified as low-excitation radio galaxies (LERGs), at redshift $z<0.15$, and with radio luminosity at $1.4 \mathrm{GHz}\left(L_{1.4}\right)$ in the range $\sim 10^{39.5}-10^{41.3} \mathrm{erg} \mathrm{s}^{-1}$. On the other hand, the FRIICAT is composed of 122 edgebrightened radio sources within the same redshift range and 
with $L_{1.4} \sim 10^{39.5}-10^{42.5} \mathrm{erg} \mathrm{s}^{-1}$. A large fraction $(\sim 90 \%)$ of the FR IIs listed are LERGs with the same type of host galaxies as FR Is. The remaining $\sim 10 \%$ show optical spectra typical of highexcitation radio galaxies (HERGs), and their hosts are bluer in the optical band and redder in the mid-IR than FR II LERGs.

Here, we report on a study we performed that is similar to that carried out for FRICAT and FRIICAT, by searching for radio galaxies with a classical FR II morphology at $1.4 \mathrm{GHz}$, but showing a projected linear size smaller than $60 \mathrm{kpc}$. From this study, we expect to find young radio sources classified as gigahertz peaked-spectrum (GPS) as well as compact steep-spectrum (CSS) sources. GPSs have typical sizes smaller than $\sim 1 \mathrm{kpc}$ and their radio spectra peak between $500 \mathrm{MHz}$ and $10 \mathrm{GHz}$ in the observer's frame, while CSSs are larger (between 1 and $20 \mathrm{kpc}$ ) and with radio spectra peaking at lower frequencies $(<500 \mathrm{MHz})$. Both classes include extremely powerful radio galaxies at $1.4 \mathrm{GHz}$, reaching $L_{1.4} \gtrsim 10^{41} \mathrm{erg} \mathrm{s}^{-1}$ (see, e.g., O'Dea 1998, for a review).

In contrast to FR Os, GPSs and CSSs are resolved in the radio band showing a typical double-lobed structure. This is the reason underlying the morphological subclassification proposed by Readhead (1995) that distinguishes between compact symmetric objects (CSO; $<1 \mathrm{kpc}$ ), medium-sized symmetric objects (MSO; $1-15 \mathrm{kpc}$ ), and large symmetric objects (LSO; > $15 \mathrm{kpc}$ ).

Several hypotheses have been proposed in the literature to explain the relation between GPS/CSSs and large-scale radio galaxies (see, e.g., Fanti et al. 1995; O’Dea 1998). According to the most accepted scenario, GOS/CSSs are "young" versions of large-scale radio sources; therefore, GPS may evolve into CSSs, and CSSs may then evolve either into FR Is or into FR IIs.

Another popular hypothesis interprets the small sizes of GPSs and CSSs by assuming that they have the same ages as large-scale radio sources, but that they have been confined by interactions with dense gas in their environment. However, observations do not support this scenario since the gas surrounding GPS/CSSs seems to be similar to that of FR II sources (Orienti \& Dallacasa 2014; see, however, Sobolewska et al. 2019).

The creation of a catalog of "small-size" FR IIs will enable us to attain a better understanding of sources with morphologies between FR 0s and FR IIs. Furthermore, such catalog could eventually let us determine whether these sources represent a completely different population of radio galaxies or whether they are young stages of the evolution of FR IIs.

The paper is organized as follows. In Sect. 2 we present the sample and the selection criteria of the catalog. The radio, optical, and infrared properties of the selected sources are described in Sect. 3. In Sect. 4 we present a comparison between the optical and radio properties of the sources, and then in Sect. 5 we present our discussion and conclusions. The tables with the properties of the selected sources and their images are collected in the Appendices A-E.

We adopt cgs units for numerical results and we also assume a flat cosmology with $H_{0}=69.6 \mathrm{~km} \mathrm{~s}^{-1} \mathrm{Mpc}^{-1}, \Omega_{\mathrm{M}}=0.286$, and $\Omega_{\Lambda}=0.714$ (Bennett et al. 2014), unless otherwise stated ${ }^{1}$. Spectral indices are based on the definition of the flux density as $S_{v} \propto v^{-\alpha}$. In general, the uncertainty on the radio luminosity is $<5 \%$, while for the linear size it is $<0.25 \mathrm{kpc}$. Therefore, we will not include error bars in our plots.

\footnotetext{
Thus, $1^{\prime \prime}$ corresponds to $2.634 \mathrm{kpc}$ at $z=0.15$.
}

\section{Sample selection}

The sources in the catalog were selected from the sample of 18286 radio sources presented by Best \& Heckman (2012; hereafter BH12) but limited to the 3357 sources with redshift $z<$ 0.15 and classified as AGN according to their reported criteria. The BH12 sample was built by cross-matching different catalogs: the optical spectroscopic catalogs based on data from the 7th Sloan Digital Sky Survey (DR7/SDSS; Abazajian et al. $2009)^{2}$ and produced by the group from the Max Planck Institute for Astrophysics and The Johns Hopkins University (Brinchmann et al. 2004; Tremonti et al. 2004), the National Radio Astronomy Observatory Very Large Array Sky Survey (NVSS; Condon et al. 1998), and the Faint Images of the Radio Sky at Twenty centimeters survey (FIRST; Becker et al. 1995). In BH12 a flux density threshold of $5 \mathrm{mJy}$ was chosen.

Source selection was based on visual examination of FIRST images carried out for all objects. Radio contours at $1.4 \mathrm{GHz}$ were built starting at a surface brightness level of $0.45 \mathrm{mJy}$ beam $^{-1}$, approximately three times the typical rms of the FIRST images for objects at $z=0.15$. This minimum surface brightness level was increased by a factor $[(1+0.15) /(1+z)]^{4}$ for closer objects to compensate for its cosmological dimming. This level corresponds to a correction factor of $\sim 1.75$ for $z=0$. We also applied a $K$ correction by assuming a spectral index of 0.7 between $178 \mathrm{MHz}$ and $1.4 \mathrm{GHz}\left(\alpha_{0.178-1.4}\right)$, as done by Schoenmakers et al. (2000) and Capetti et al. (2017b). Overall this correction was rather small, being $\sim 10 \%$.

Selected sources were those displaying radio emission at the sensitivity limit of the FIRST images within a circle of $30 \mathrm{kpc}$ radius centered on the optical position. This size is large enough to ensure that the radio emission is still contained within the host galaxy. We selected only radio sources with a FRIIlike morphology (i.e., edge-brightened) as done in Capetti et al. (2017a). Thus, by applying the cut of having extended radio structure with projected size less than $60 \mathrm{kpc}$ and selecting sources with FRII radio morphology, we considered those that were excluded from the FRIICAT. Five collaborators carried out the morphological classification independently, and only sources selected by at least three of them were included in the final catalog.

We called "compact doubles" the FR II radio sources with radio emission contained in the host galaxy and having two peaks of surface brightness; therefore, our catalog was called COMPact Doubles CATalog (COMP2CAT). The term compact double was first coined by Phillips \& Mutel (1982), although they called "symmetric compact doubles" sources with the same radio morphology as those we selected, but with projected linear sizes $\leq 1 \mathrm{kpc}$. Since the selection carried out was based on morphology, COMP2CAT is analogous to a CSO/MSO/LSO sample with projected linear sizes limited to $60 \mathrm{kpc}$.

Following our definition, we selected 78 sources from the original sample. However, there were cases of selected sources having one of the peaks of the radio surface brightness closer to the SDSS optical position than the other. These sources could be, for example, chance alignments of unrelated sources (i.e., background or foreground objects), close counterparts, or FRI-like sources instead of true compact doubles. To distinguish between these cases, we defined the asymmetric index $A$,

$A=\frac{\left|r_{2}-r_{1}\right|}{r_{2}+r_{1}}$ 


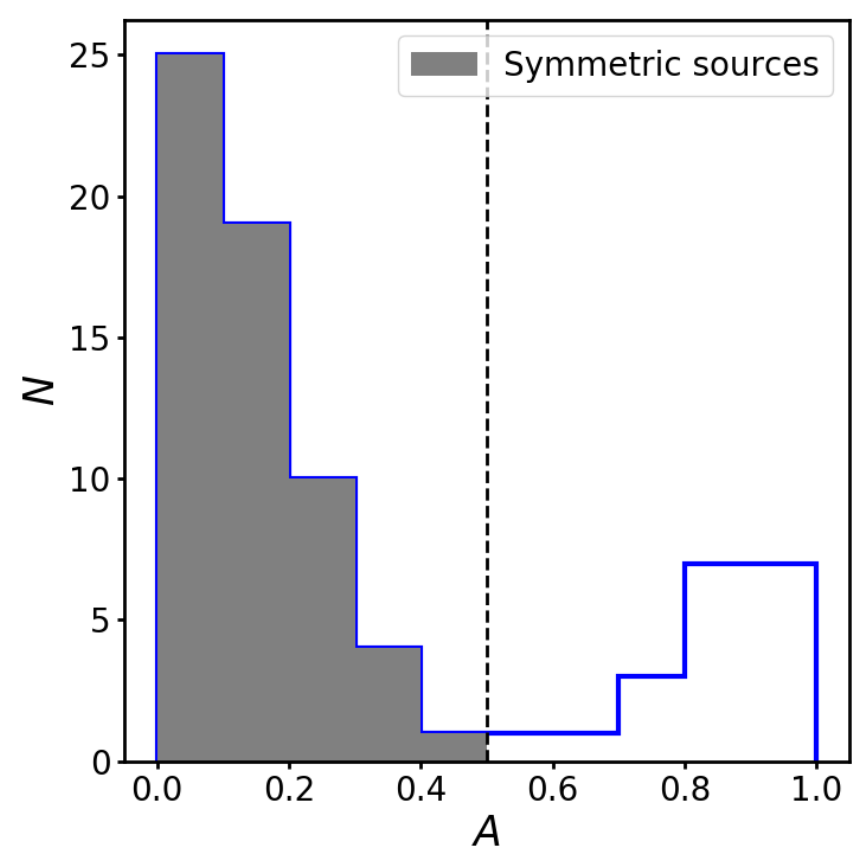

Fig. 1. Asymmetric index distribution of the 78 sources initially selected as defined in Eq. (1). The black vertical dashed line indicates the separation between symmetric and asymmetric sources defined as $A=0.5$. Symmetric sources are those with $A \leq 0.5$.

where $r_{1}$ and $r_{2}$ are the distances of each radio surface brightness peak from the optical position. The $A$ parameter ranges between 0 and 1: symmetric sources display $A \sim 0$, whereas $A=1$ corresponds to extremely asymmetric sources. Figure 1 represents the distribution of the asymmetric indices for all the selected sources. Given that the bi-modal distribution peaks around $A=$ 0.1 (i.e., more symmetric sources) and around $A=0.9$ (i.e., very asymmetric sources), and since we wanted to focus on the most symmetric sources, we cut out those with $A \geq 0.5$, which corresponds to one of the peaks of surface brightness being at a distance from the optical position three times larger than the other peak. A comparison between symmetric and asymmetric sources is shown in Fig. 2, which shows an example of a COMP2CAT source identified as symmetric using our criterion (left panel), and two of the sources identified as asymmetric (central and right panels). As shown in Fig. 2, this definition of asymmetric index enabled us to exclude double sources with asymmetric radio morphology.

We also considered a few more exclusions based on radio and optical properties:

1. Sources with unclear radio classification based on highresolution radio maps obtained from the National Radio Astronomy Observatory (NRAO) Very Large Array (VLA) archive $^{3}$ (see Appendix A): SDSS J111025.09+032138.8, SDSS J125724.35+272952.1, and SDSS J132451.44+362242.7 (using radio maps at $1.4 \mathrm{GHz}$ with resolutions of 12.40 , 1.43 , and 1.31 arcsec, respectively) and SDSSJ125935.70+ 275733.3 and SDSS J161531.36+272657.3 (using radio maps at $5 \mathrm{GHz}$ with resolutions of 1.51 and $1.18 \mathrm{arcsec}$ ).

2. "Restarted" radio sources (or double-double radio galaxies; see Schoenmakers et al. 2000 and Appendix B) based on their extended $1.4 \mathrm{GHz}$ and $150 \mathrm{MHz}$ emission: SDSS
J152804.95+054428.1, SDSS J132345.01+313356.7, SDSS J215305.08-071106.9， SDSS J115905.68+582035.5， and SDSS J083830.99+194820.4

3. Sources with a FRI-like morphology at scales of hundreds of kiloparsecs (see Capetti et al. 2017b and Appendix B) based on their extended $1.4 \mathrm{GHz}$ and $150 \mathrm{MHz}$ emission: SDSS J091443.12+073554.9 and SDSS J083224.82+184855.4

After finalizing the selection, COMP2CAT includes 43 sources whose properties and contours from FIRST (at $1.4 \mathrm{GHz}$ with a resolution of $5 \operatorname{arcsec}$ ), NVSS (at $1.4 \mathrm{GHz}$ with a resolution of $45 \mathrm{arcsec}$ ), and the Tata Institute of Fundamental Research (TIFR) Giant Metrewave Radio Telescope (GMRT) Sky Survey (TGSS; at $150 \mathrm{MHz}$ with a resolution $>25$ arcsec) are represented in Table C.1 and in Fig. D.1. We used FIRST radio maps to carry out the morphological identification of sources while obtaining the $1.4 \mathrm{GHz}$ fluxes from NVSS. We chose to use the $1.4 \mathrm{GHz}$ fluxes from NVSS instead of those from FIRST because FIRST could have missed some of the flux from largescale structures due to its lack of short baselines.

Other catalogs of compact radio galaxies, with sources mainly selected on the basis of their radio properties, are present in the literature. In particular, Snellen et al. (2004) published the CORALZ catalog of compact radio sources at low redshifts. This catalog was built by selecting those sources in FIRST with an optical counterpart in the APM Palomar Sky Survey (APM/POSS-I) catalog and radio flux densities at $1.4 \mathrm{GHz}$ $>100 \mathrm{mJy}$ and angular sizes $<2 \operatorname{arcsec}$ (which translates into a projected linear size $<5.6 \mathrm{kpc}$ using our cosmology). Following these criteria, the CORALZ catalog is made of 28 sources at $0.005<z<0.16$, of which 17 form a $95 \%$ statistically complete sample.

On the other hand, for COMP2CAT we did not impose any limits on the radio flux density of the sources and we included sources with projected linear sizes up to $60 \mathrm{kpc}$; thus, we expected to find physically larger and less radio luminous sources in COMP2CAT than those in the CORALZ catalog.

\section{COMP2CAT host and radio properties}

\subsection{Radio properties}

COMP2CAT sources appear as the low radio luminosity tail of FR Is and FR IIs, with a distribution of NVSS $1.4 \mathrm{GHz}$ radio luminosities that ranges in the interval $10^{38.5} \lesssim L_{1.4} \lesssim 10^{41} \mathrm{erg} \mathrm{s}^{-1}$ and peaks around $\sim 10^{39.8} \mathrm{erg} \mathrm{s}^{-1}$, as shown in Fig. 3. This figure also shows the separation between FR Is and FR IIs established by Fanaroff \& Riley (1974), $L_{0.178} \sim 2.8 \times 10^{41} \mathrm{erg} \mathrm{s}^{-1} \mathrm{sr}^{-1}$, which we adapted to the cosmology chosen here and assuming $\alpha_{0.178-1.4}=0.7\left(L_{1.4} \sim 10^{41.6} \mathrm{erg} \mathrm{s}^{-1}\right)$. Most objects included in COMP2CAT, FRICAT, and FRIICAT fall below this threshold.

The left panel of Fig. 4 shows the projected radio linear size distribution of COMP2CAT sources. Their projected radio linear sizes range from 5 to $45 \mathrm{kpc}$, peaking around $15 \mathrm{kpc}$ and correspond to the distances between the two peaks of radio surface brightness at $1.4 \mathrm{GHz}$. Thus, according to the morphological classification for compact doubles presented by Readhead (1995), COMP2CAT includes 11 MSOs and 32 LSOs.

The spectral index between $150 \mathrm{MHz}$ and $1.4 \mathrm{GHz}, \alpha_{0.15-1.4}$, was computed as follows:

$\alpha_{0.15-1.4}=-1.03 \log \left(\frac{S_{1.4}}{S_{0.15}}\right)$

\footnotetext{
3 Available at: http://wWw.aoc.nrao.edu/ vlbacald/ ArchIndex.shtml
} 

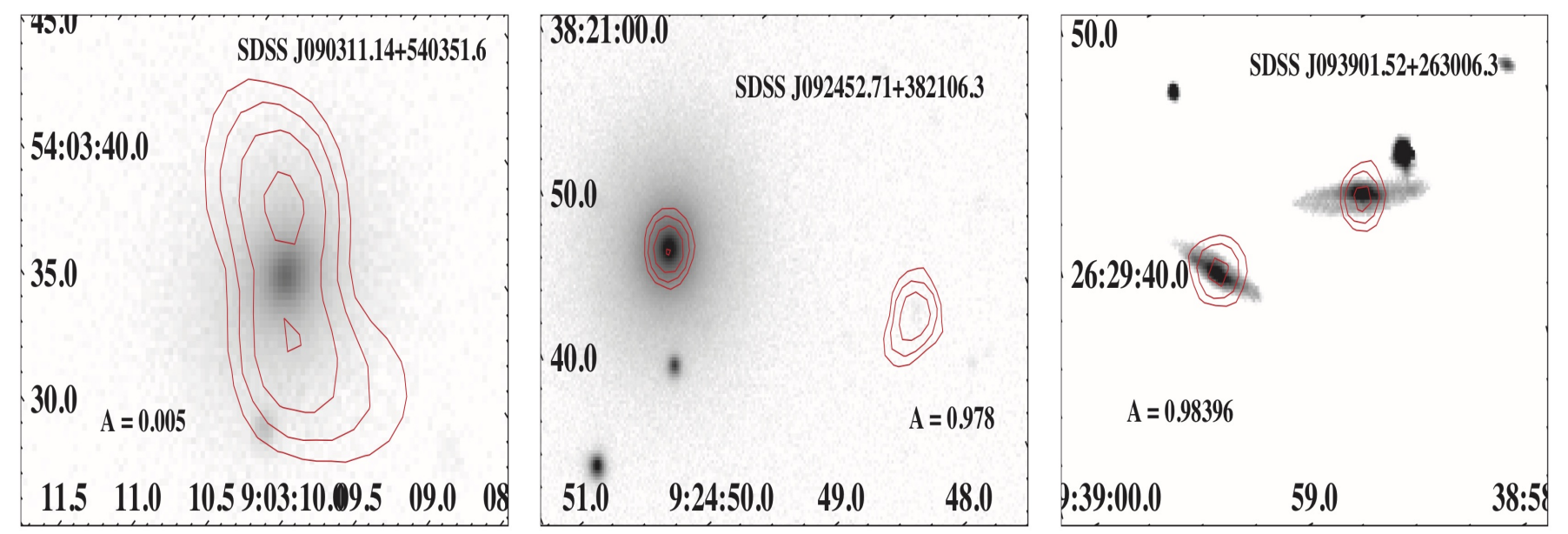

Fig. 2. $r$-band SDSS images with FIRST contours superimposed for a symmetric (left) and two asymmetric (middle and right) sources, according to their FIRST contours. Left: contours start at $2 \mathrm{mJy} \mathrm{beam}^{-1}$ and increase by a factor of 2 . Middle: contours start at $0.6 \mathrm{mJy}^{-1}$ beam ${ }^{-1}$ and increase by a factor of 2. Right: contours start at $0.5 \mathrm{mJy}^{\text {beam }}{ }^{-1}$ and increase by a factor of 2 .

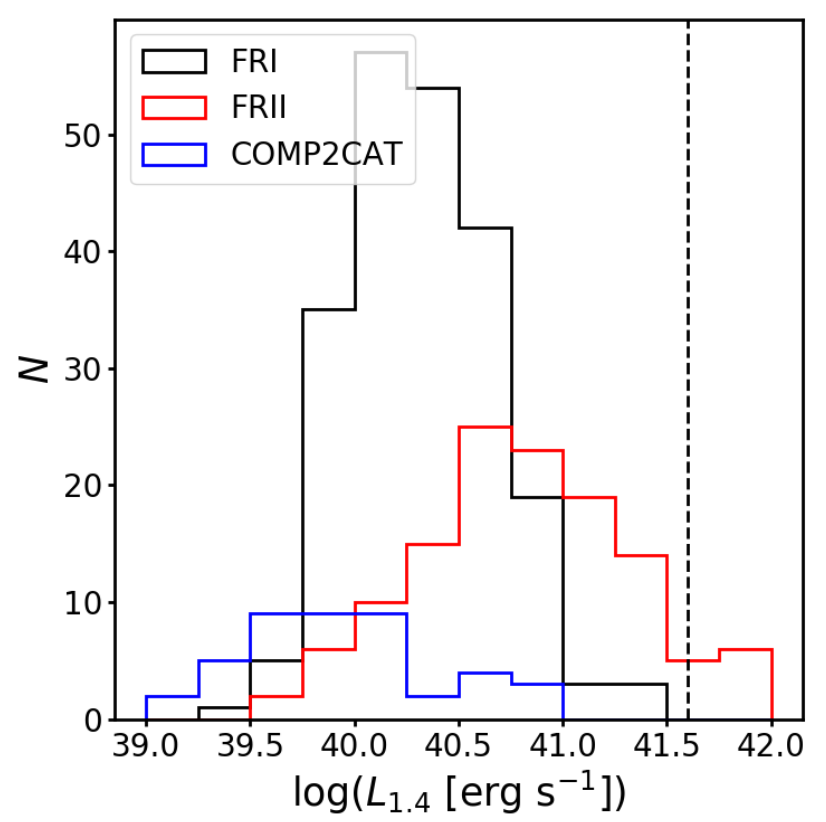

Fig. 3. Radio luminosity distribution of COMP2CAT, FRICAT, and FRIICAT (blue, black, and red dots, respectively). The dashed vertical line indicates the transition power between FR I and FR II reported by Fanaroff \& Riley (1974).

where $S_{1.4}$ is the NVSS flux density and $S_{0.15}$ the TGSS flux density.

We show the distribution of spectral indices in the right panel of Fig. 4. The bulk of COMP2CAT sources have $0.3 \leq$ $\alpha_{0.15-1.4} \leq 0.9$ and their distribution peaks around $\alpha_{0.15-1.4}=$ 0.5 , while only eight COMP2CAT sources do not have TGSS counterparts, and thus their spectral indices could not be estimated.

Labiano (2006) defined CSSs as those sources with linear sizes smaller than $15 \mathrm{kpc}$ and $\alpha>0.5$, while according to a more recent analysis of Orienti \& Dallacasa (2014) this definition could be extended to sources with linear sizes smaller than $20 \mathrm{kpc}$ and $\alpha>0.7$. In Fig. 5, $\alpha_{0.15-1.4}$ is represented against the projected linear size. Thus, possible CSSs in the COMP2CAT are those lying in the upper left corner of Fig. 5, whereas the previous definitions are corrected for the adopted cosmological parameters (see Sect. 1). According to the criteria adopted by Labiano (2006), there are seven possible CSSs in COMP2CAT (SDSS J073600.87+273926.0, SDSS J074641.45+184405.4, SDSS J090311.14+540351.6, SDSS J111109.58+393552.0, SDSS J113643.49+545446.8， SDSS J144731.24+330606.2, and SDSS J164452.86+341251.3), while according to the definition by Orienti \& Dallacasa (2014), only one of the COMP2CAT sources, SDSS J113305.52+592013.7, can be considered a CSS. Therefore, adopting Orienti \& Dallacasa (2014) criteria, CSSs do not constitute an important fraction of COMP2CAT.

Additionally, we estimated the spectral index between 1.4 and $5 \mathrm{GHz}$ using the Green Bank $6 \mathrm{~cm}$ (GB6) Radio Source Catalog and the NVSS flux. Only 14 COMP2CAT sources have GB6 counterparts. Furthermore, the full width at half maximum (FWHM) of the primary beam of GB6 is $\sim 3.5$ arcmin; thus, the fluxes obtained at $5 \mathrm{GHz}$ are only upper limits since there are multiple FIRST sources inside the beam that could contaminate the result. We checked the sources individually and found that the only ones that could have important contamination from neighbor sources are SDSS J132649.30+164948.0, SDSS J135338.43+360802.4, and SDSS J155749.61+161836.6. In these cases, the spectral indices obtained are regarded as lower limits.

The comparison between the spectral indices at low and high frequencies is shown in Fig. 6. The distribution of $\alpha_{1.4-5}$ ranges from 0.2 to 0.9 and peaks around $\alpha_{1.4-5}=0.65$. Five COMP2CAT sources are actually out of GB6 footprint, while the flux at $5 \mathrm{GHz}$ of the remaining sources of the sample, assuming either a flat spectrum or the same spectral index as from $150 \mathrm{MHz}$ to $1.4 \mathrm{GHz}$, is below the completeness level of GB6 (50 mJy); the only exceptions are (i) SDSS J125935.70+275733.3 and (ii) SDSS J091134.75+125538.1. We were able to estimate lower limits for their spectral indices between 1.4 and $5 \mathrm{GHz}$ : (i) $\alpha_{1.4-5}>0.56$ and (ii) $\alpha_{1.4-5}>0.98$.

Finally, we show a comparison between $L_{1.4}$ and the projected linear size in Fig. 7. If COMP2CAT sources were the predecessors of FRIICAT sources, we would see an increase in the luminosity with size. However, we do not see a clear trend. This could indicate either that these sources could evolve into lowluminosity FRIIs or that they represent a different population of radio sources. 

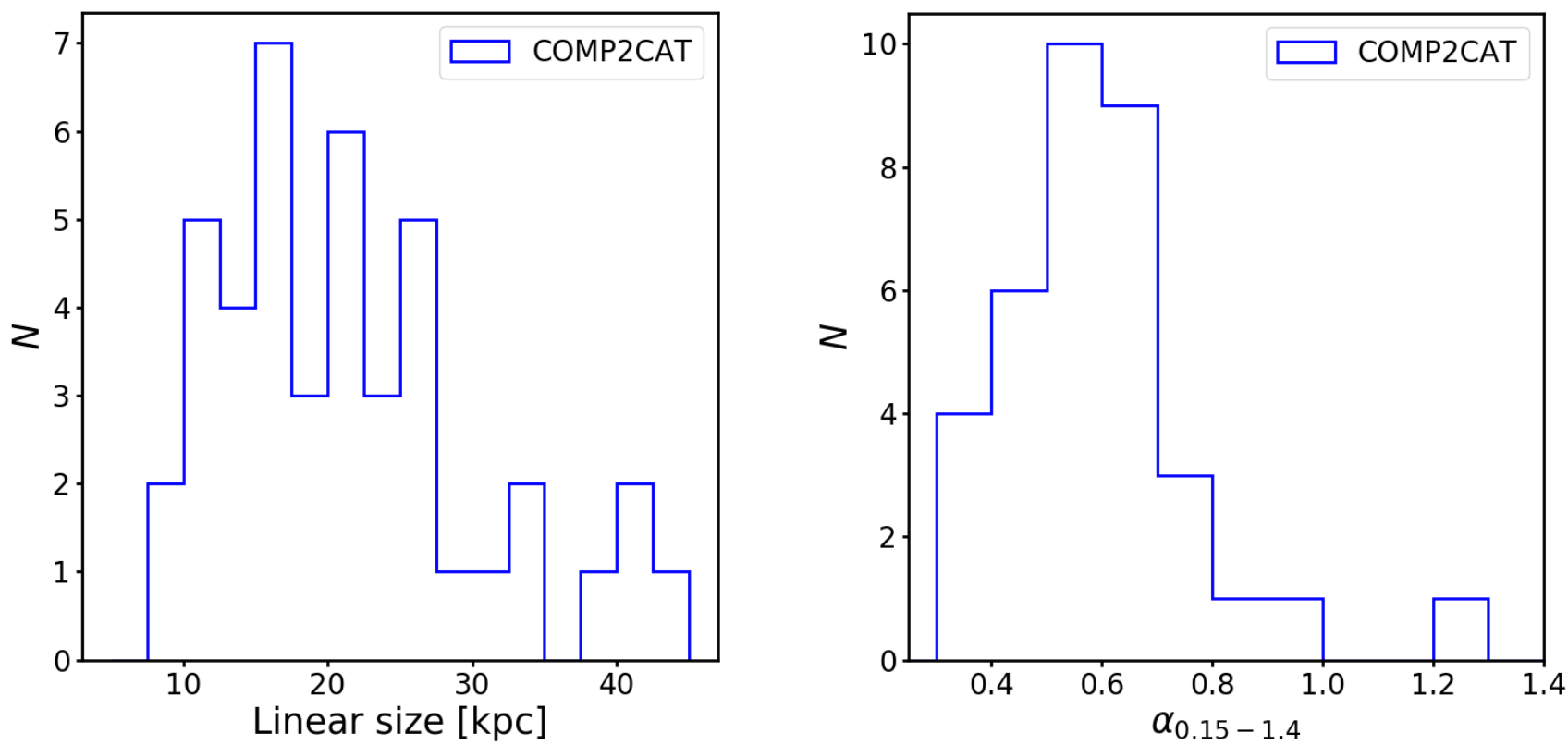

Fig. 4. Left: projected linear sizes distribution of COMP2CAT sources. Right: distribution of the spectral index between $150 \mathrm{MHz}$ and $1.4 \mathrm{GHz}$ for sources in COMP2CAT. This spectral index was obtained using 1400 and $150 \mathrm{MHz}$ fluxes from NVSS and TGSS, as shown in Eq. (2).

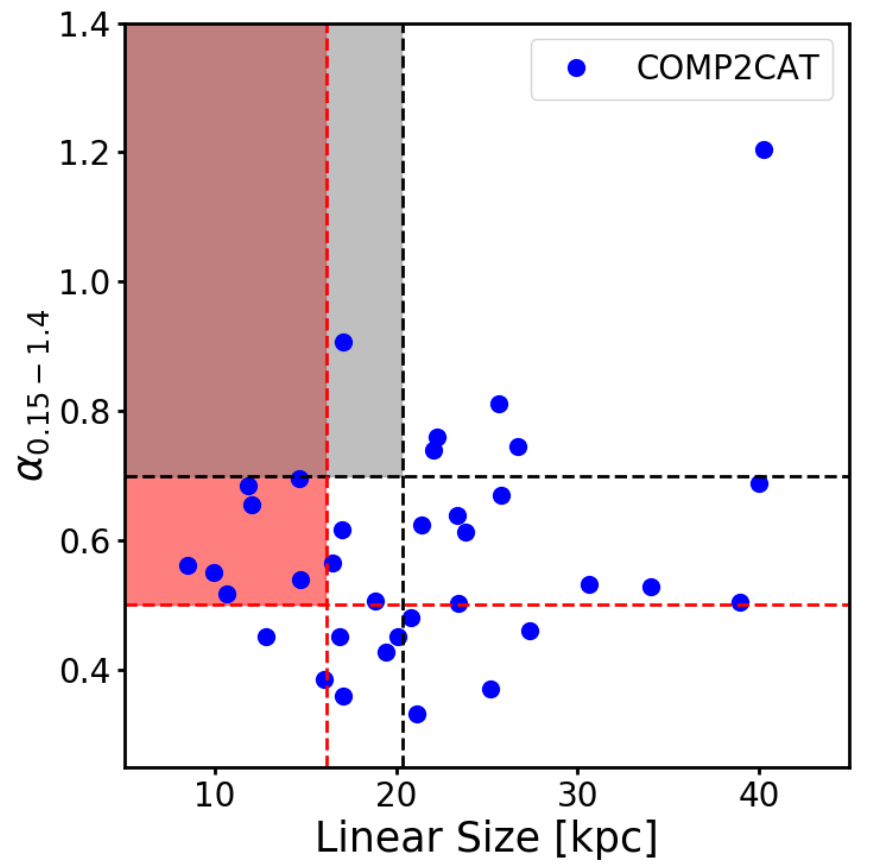

Fig. 5. Spectral index between $150 \mathrm{MHz}$ and $1.4 \mathrm{GHz}$ vs. linear size of COMP2CAT sources. The red lines mark the criteria chosen by Labiano (2006) to define CSSs: projected linear sizes below $15 \mathrm{kpc}$ and $\alpha_{0.15-1.4} \gtrsim 0.5$ (corrected using the cosmology adopted). The area shaded in red corresponds to sources that would be considered CSSs according to these criteria. The black lines correspond to the criteria presented by Orienti \& Dallacasa (2014): projected linear sizes smaller than $20 \mathrm{kpc}$ and $\alpha_{0.15-1.4} \gtrsim 0.7$ (also corrected using our cosmology). The area shaded in gray marks the CSS area in the diagram based on the Orienti \& Dallacasa (2014) selection.

Images with the radio contours for COMP2CAT sources are represented in Appendix D (see Fig. D.1). For each source,

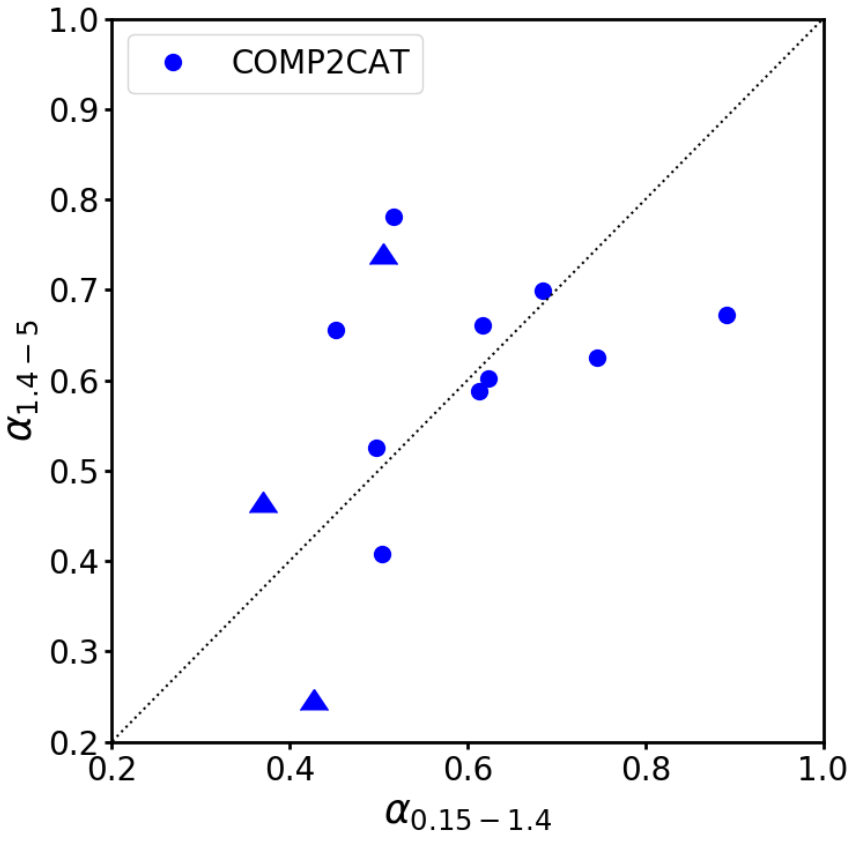

Fig. 6. Distribution of spectral indices between 1.4 and $5 \mathrm{GHz}$ for COMP2CAT sources with GB6 counterparts. Upward arrows indicate lower limits.

we show the FIRST ${ }^{4}$ (black), the $\mathrm{NVSS}^{5}$ (red), and the TGSS (blue) contours. The contours at a given frequency are drawn by choosing a starting surface brightness level and increasing this value by a chosen factor. The starting level and the increase factor for each source and frequency are listed in Table E.3.

\footnotetext{
4 Available at https://third.ucllnl.org/cgi-bin/ firstcutout

5 Available at https://www.cv.nrao.edu/nvss/postage. shtml

6 Available at https://vo.astron.nl/tgssadr/q_fits/imgs/ form
} 


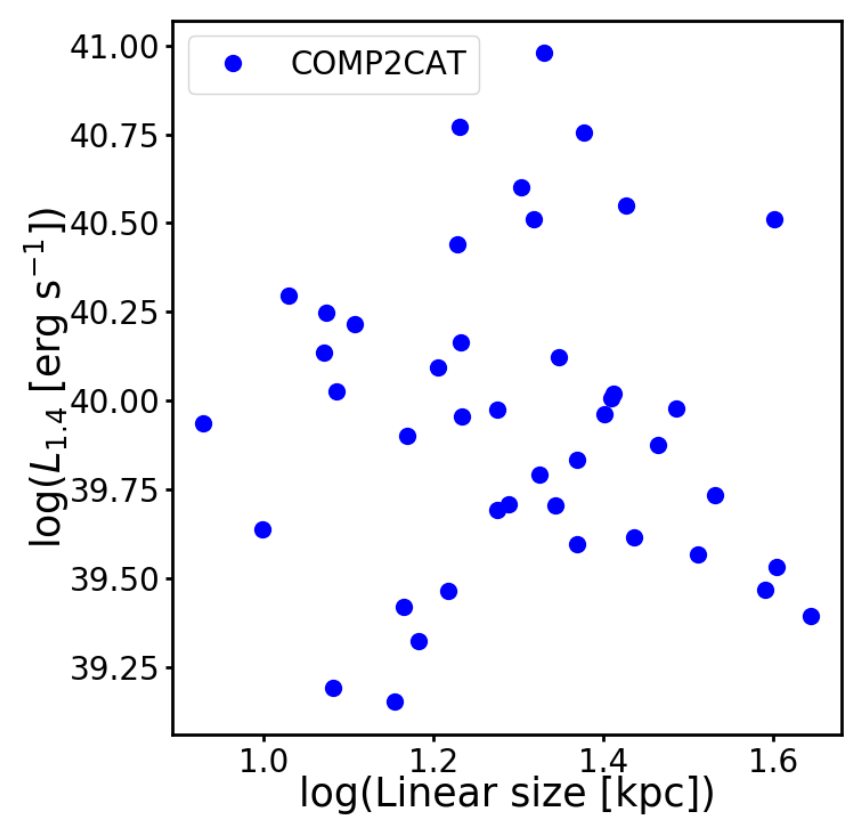

Fig. 7. Radio luminosities at $1.4 \mathrm{GHz}$ as a function of projected linear sizes of COMP2CAT sources.

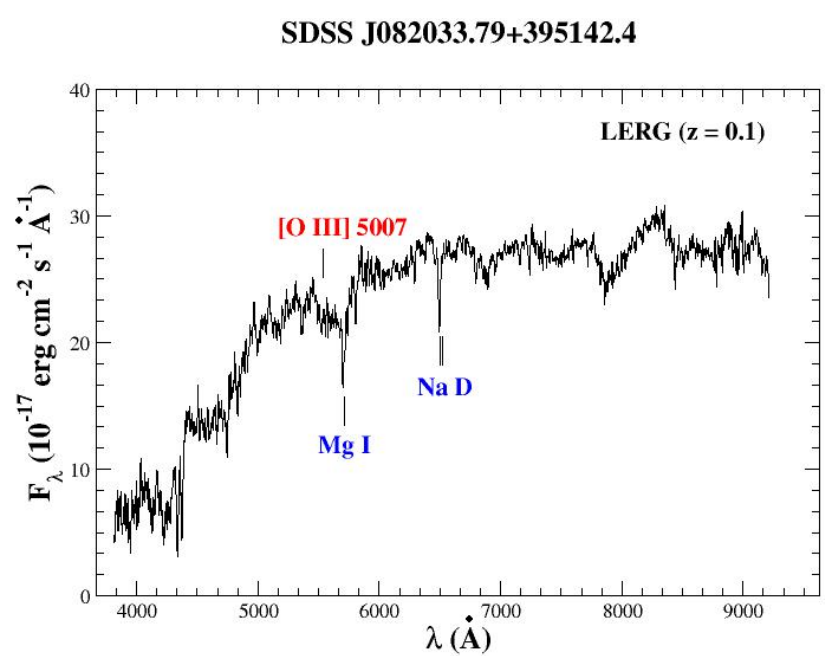

Fig. 8. Optical spectrum of SDSS J082033.79+395142.4, selected in COMP2CAT, from $4000 \AA$ and $9000 \AA$ available in the SDSS database. This source is a clear LERG. Optical emission (red) and absorption (blue) lines identified are indicated in the figure.

\subsection{Optical and infrared properties}

All COMP2CAT sources are classified as LERGs; the only exception is SDSS J101653.82+002857.0, which is a possible HERG. Differences in the spectra of LERGs, HERGs, and starforming galaxies are represented in Figs. 8-10. Baldi \& Capetti (2010) claimed that there is contamination of $\sim 10 \%$ from radio-quiet AGN in the SDSS/NVSS sample. In our case, the contamination would mainly come from Seyfert galaxies, since we do not expect other radio-quiet galaxies to form double structures with sizes exceeding a few kiloparsecs. Actually, we only found one object with strong optical emission features that could be either a Seyfert galaxy or a HERG; therefore, the contamination from radio-quiet AGN is negligible in our case.

We obtained the equivalent width of the [O III] $\left(E W_{\text {[OIIII] }}\right)$ from the SDSS database, and show its distribution on Fig. 11.
SDSS J101653.82+002857.0

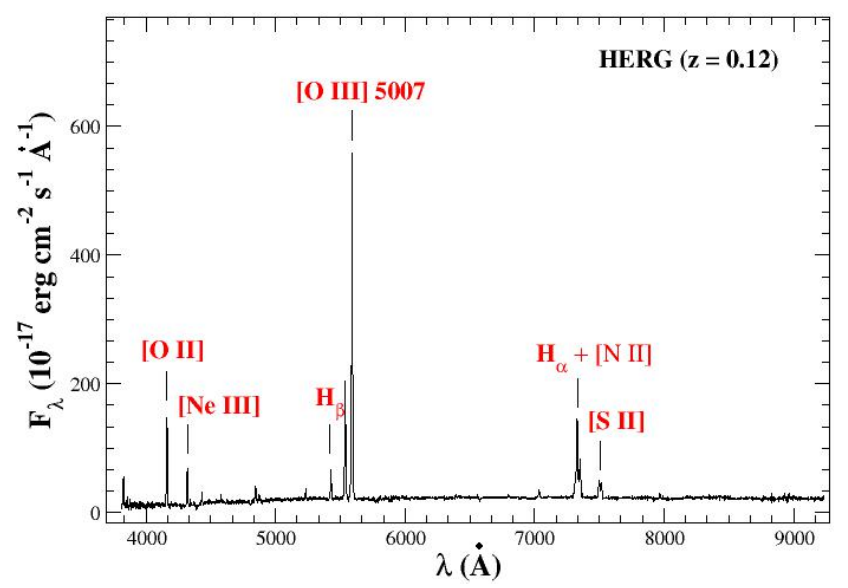

Fig. 9. Same as Fig. 8, but for SDSS J101653.82+002857.0, the only HERG belonging to COMP2CAT .

\section{SDSS J111025.09+032138.8}

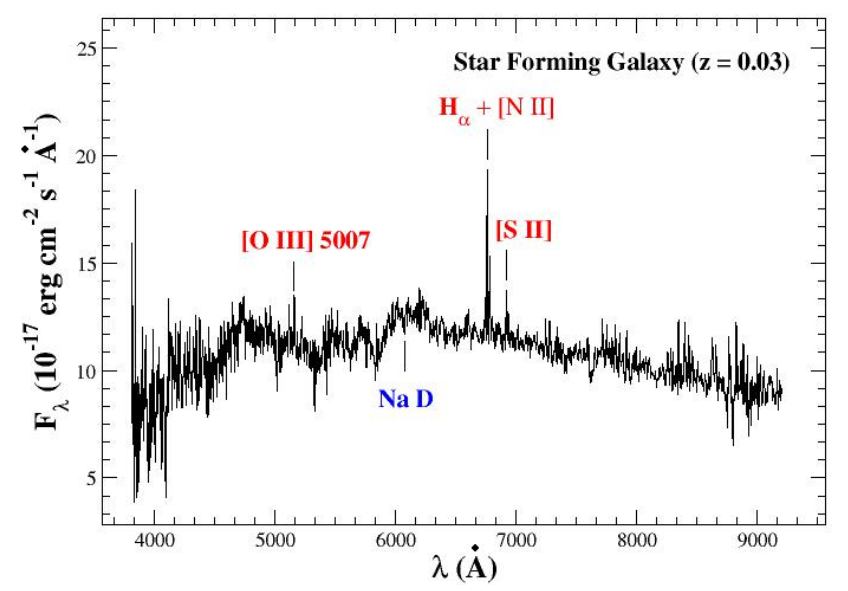

Fig. 10. Same as Fig. 8, but for the case of SDSS J111025.09+032128.8, the star-forming galaxy excluded from our final selection on the basis of its radio morphology shown in the VLA radio image.

The HERG source has not been included in this plot due to its high $E W_{\text {[OIII] }}$ value ${ }^{7}$. The $E W_{\text {[OIII] }}$ values range between $0.5 \lesssim E W_{[\mathrm{OIII}]} \lesssim 3 \AA$ and peak at $E W_{\text {[OIII] }} \sim 0.5 \AA$. According to Capetti \& Baldi (2011) stellar processes (instead of the AGN) can dominate the [O III] line emission, especially for low radio luminosity sources, and this can be distinguished on the basis of the $E W_{[\mathrm{OIII}]}$ in such a way that the [O III] line emission from COMP2CAT sources presenting $E W_{\text {[OIII] }} \leq 1.7 \AA$ is mostly due to stellar processes. This is the case for $\sim 80 \%$ of COMP2CAT sources, as we will show in the following section. Even considering this effect, we found that COMP2CAT sources have [O III] luminosities $\left(L_{[\mathrm{OIII}]}\right)$ thousands of times smaller than the values that Labiano (2009) found for GPSs and CSSs, which highlights that sources in COMP2CAT constitute a different population than GPS/CSSs, as already shown in Fig. 5.

The left panel of Fig. 12 shows the redshift distribution of COMP2CAT in comparison with the same distribution for FRICAT and FRIICAT. The COMP2CAT redshift distribution appears rather flat. As can be seen in the right panel of Fig. 12,

\footnotetext{
7 SDSS J101653.82+002857.0: $E W_{\text {[OIII] }} \sim 190 \AA$.
} 


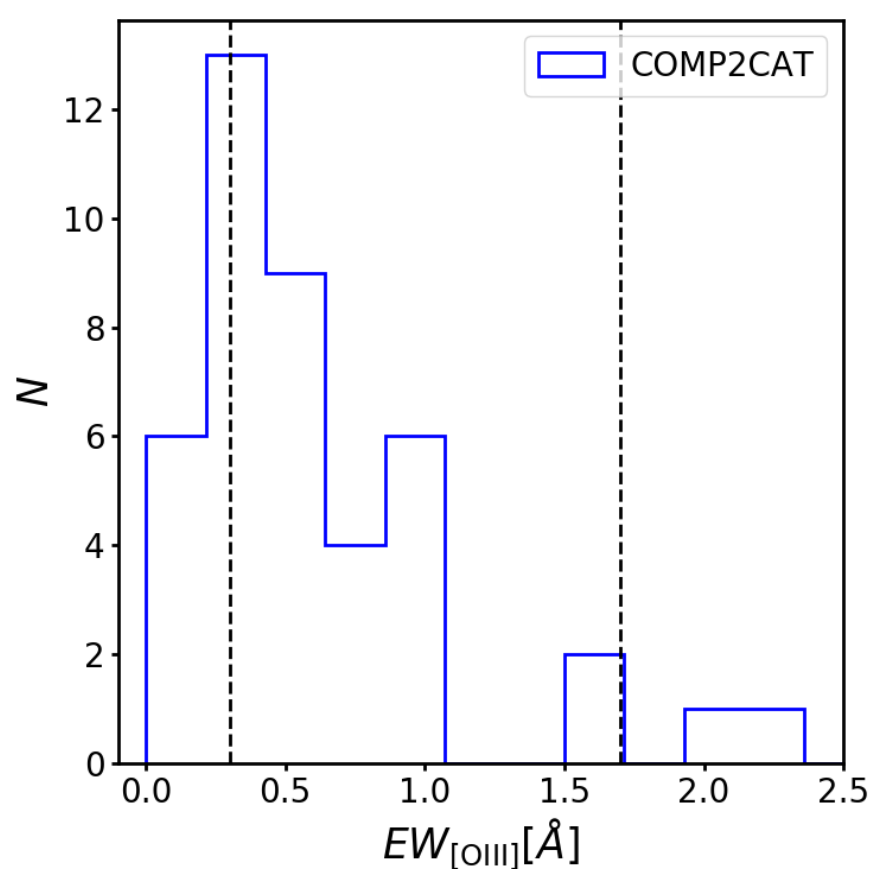

Fig. 11. Distribution of equivalent widths of the [O III] line for COMP2CAT sources. The black vertical dashed lines limit the region where $L_{[\mathrm{OIII}]}$ is dominated by populations of old stars instead of by the AGN according to Capetti \& Baldi (2011).

we binned the redshift distribution and fitted it as $N \propto z^{3}$, leaving out the higher redshift tail of the distribution, i.e., the last bin. In this way, we expect $\sim 91$ sources in the whole redshift range. However, we actually observed only $47 \%$ of them, so COMP2CAT is only $\sim 47 \%$ complete at $z<0.15$.

A possible, simple explanation is that the remaining sources are lost due to their small sizes, their faint radio luminosities, or a combination of these two effects. To estimate the number of sources potentially lost, we took the radio luminosities and linear sizes of the subsample of COMP2CAT sources at $0.04 \leq z \leq 0.08$ and assigned a random value of redshift in the range $0.11 \leq z \leq 0.15$ to each of them. We computed their radio fluxes and angular sizes at the new redshifts and checked how many of them fall below the sensitivity limit $(5 \mathrm{mJy})$ and angular resolution ( $5 \mathrm{arcsec}$ ) of FIRST. We estimated that the low radio luminosities and the small linear sizes of the sources account for the loss of $\sim 25 \%$ and $\sim 20 \%$ of the sources between $z=0.11$ and $z=0.15$, which corresponds to a loss of $\sim 11 \%$ over the whole catalog. It is important to note that this test is highly sensitive to the number of sources taken as the low-redshift sample, due to the poor number of sources in the lower redshift range.

Out of the $42 \%$ of lost sources remaining, at least a $10 \%$ can be explained by the incompleteness of the SDSS because, according to Strauss et al. (2002) and Montero-Dorta \& Prada (2009), the SDSS is complete up to $\sim 90 \%$ for apparent magnitudes in the range $14.5<r<17.77$. This incompleteness is mostly due to the SDSS fiber collision, that does not allow us to place the fibers closer than 55" apart. The apparent magnitude distribution of COMP2CAT sources ranges from 12.5 to $18 \mathrm{mag}$, peaking at $15 \mathrm{mag}$; there is only one source with $r>17.7$ and six with $r \leq 14.5$. The $\sim 32 \%$ of loss left is consistent with the uncertainties of our analysis and the possible non-uniform selections performed.

In order to determine the completeness level of our catalog at lower redshift, namely at $z<0.1$, we binned the redshift distribution up to $z=0.1$ and we fitted it leaving out the last bin, as previously done. Thus, our catalog is $\sim 83 \%$ complete up to $z=0.1$. Nevertheless, due to the low number of sources at $z<0.1$, this analysis is not statistically significant.

Hosts galaxies of COMP2CAT sources show a distribution of absolute magnitudes in the $r$ band $\left(M_{r}\right)$ and of black hole masses $\left(M_{\mathrm{BH}}\right)$ (Fig. 13, left and right panels, respectively), similar to FRICAT and FRIICAT sources. The $M_{r}$ ranges from -21 to -24 and peaks at $M_{r} \sim-22.5$, whereas the black hole mass is in the range of $7.5 \lesssim \log M_{\mathrm{BH}} \lesssim 9.5 M_{\odot}$, peaking at $\sim 10^{8.5} M_{\odot}$.

The value of $M_{\mathrm{BH}}$ was computed using its correlation with the stellar velocity dispersion, $\sigma_{*}$, published by Tremaine et al. (2002):

$\log \left(M_{\mathrm{BH}} / M_{\odot}\right)=\alpha+\beta \log \left(\sigma_{*} / \sigma_{0}\right)$,

with $\alpha=8.13 \pm 0.06, \beta=4.02 \pm 0.32$, and $\sigma_{0}=200 \mathrm{~km} \mathrm{~s}^{-1}$. The error on $M_{\mathrm{BH}}$ is dominated by the spread of the relation, so the $M_{\mathrm{BH}}$ presented have an uncertainty of a factor of $\sim 2$.

As previously performed for FRICAT and FRIICAT, we computed the concentration index, $C_{r}$, defined as the ratio of the radii including $90 \%$ and $50 \%$ of the light in the $r$ band, respectively. This index tends to have higher values $(\gtrsim 2.86$, according to Nakamura et al. 2003; Shen et al. 2003, or $\gtrsim 2.6$, according to Strateva et al. 2001; Kauffmann et al. 2003; Bell et al. 2003) for early-type galaxies (ETGs) than for late-type galaxies.

In addition, we also estimated the $\operatorname{Dn}(4000)$ index, defined as the ratio of the flux density on the red side of the Ca-II break (4000-4100 $\AA$ ) to that on the blue side (3850-3950 $)$ (Balogh et al. 1999). The $\operatorname{Dn}(4000)$ index is lower in the presence of young stars and non-stellar emission; according to Capetti \& Raiteri (2015), red galaxies at $z<0.15$ have $\operatorname{Dn}(4000)=1.95 \pm 0.05$.

The left panel of Fig. 14 (where the Dn(4000) index versus the $C_{r}$ index is represented for the sources in the three catalogs) shows that most of the sources in COMP2CAT are ETGs since they present high values of both indices. In the same figure, the right panel shows $C_{r}$ versus the $M_{\mathrm{BH}}$. This plot shows no change in $M_{\mathrm{BH}}$ with $C_{r}$.

We also show the $u-r$ color of the host galaxy versus its $M_{r}$ (Fig. 15, left panel) since the $u-r$ color gives information on the properties of the whole source, while the Dn(4000) index only gives information about the region of $3^{\prime \prime}$ in diameter covered by the SDSS spectroscopic aperture. The $u-r$ color was not corrected for galactic extinction since the correction is $\leq 5 \%$. We see that most of the COMP2CAT sources are in the region of red ETGs (Schawinski et al. 2009). The only source in our sample that is not an ETG according to this diagnostic is the HERG.

As previously carried out for the other radio galaxy catalogs we also checked the mid-IR colors of selected COMP2CAT sources. To obtain the WISE magnitudes of the sources in COMP2CAT, we associated the position of their sources adopting a 3 ".3 angular separation, which corresponds to the combination of the typical positional uncertainty of the WISE all-sky survey (Wright et al. 2010) and that of the FIRST (D'Abrusco et al. 2014; Massaro et al. 2014).

The WIS E magnitudes in the [3.4], [4.6], [12], and [22] $\mu \mathrm{m}$ nominal filters ( $W 1, W 2, W 3$, and $W 4$, respectively) are in the Vega system. Their values and those of the colors derived using them have not been corrected for Galactic extinction, which can be considered negligible since it only affects to the magnitude at $3.4 \mu \mathrm{m}$ of sources at low Galactic latitudes and, even in these cases, the correction is less than $\sim 3 \%$ (D'Abrusco et al. 2014).

The right panel of Fig. 15 is a color-color plot of the COMP2CAT, FRICAT, and FRIICAT sources. In general, 

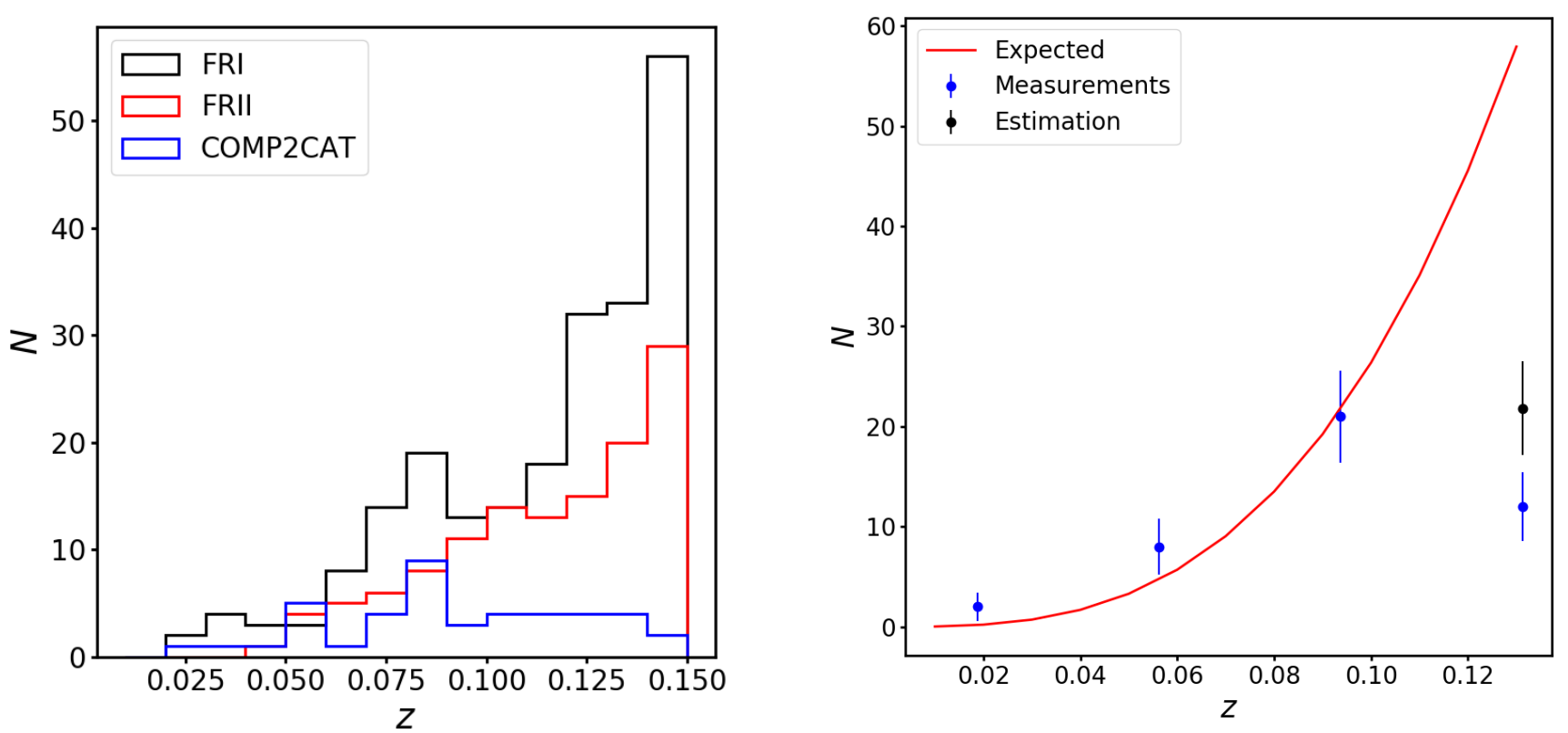

Fig. 12. Left: histograms showing the redshift distribution of COMP2CAT (blue), FRICAT (black), and FRIICAT (red) sources. Right: observed redshift distribution (blue dots) and expected redshift distribution (red line). The black dot corresponds to the number of galaxies that would be present if the only effects playing a role were the low luminosities and the small sizes of the sources.
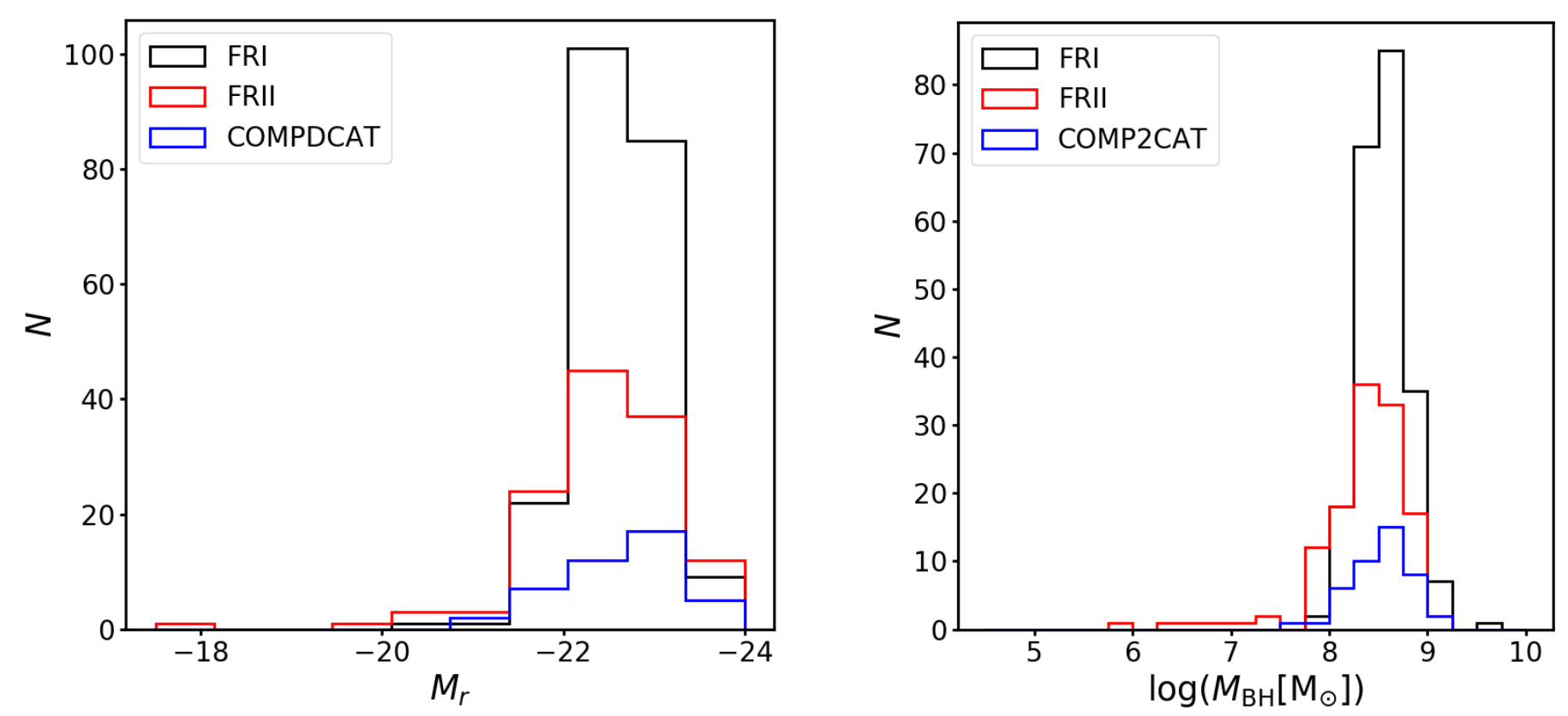

Fig. 13. Distributions of the $r$-band absolute magnitude (left) and black hole masses (right) for COMP2CAT (blue), FRICAT (black), and FRIICAT (red).

COMP2CAT sources display bluer mid-IR colors than the sources in FRICAT and FRIICAT. This could be explained by a smaller amount of dust in COMP2CAT sources. However, the W3 magnitude distributions of COMP2CAT, FRICAT, and FRIICAT sources are similar, peaking in all cases at $W 3 \sim 12$, while the COMP2CAT $W 1$ and $W 2$ magnitude distributions seem to peak at lower values ( 12.25 in both cases) than those for FRICAT and FRIICAT sources (which peak at 13.25). Nevertheless, the $W 3$ magnitudes of nine sources (between 11.8 and $12.4 \mathrm{mag}$ ) are actually upper limits, so the distribution of $W 3$ for COMP2CAT sources could show the same differences (about $\sim 8 \%$ ) with respect to FRI and FRII sources as the $W 1$ and $W 2$ magnitudes.

\section{Comparison between optical and radio luminosity}

Here we compare the multifrequency behavior of COMP2CAT sources with that of other radio galaxy catalogs.

The comparison shown in Fig. 16 of the [O III] line luminosity $\left(L_{[\mathrm{OIII}]}\right)$ to the NVSS radio luminosity at $1.4 \mathrm{GHz}\left(L_{1.4}\right)$ is 

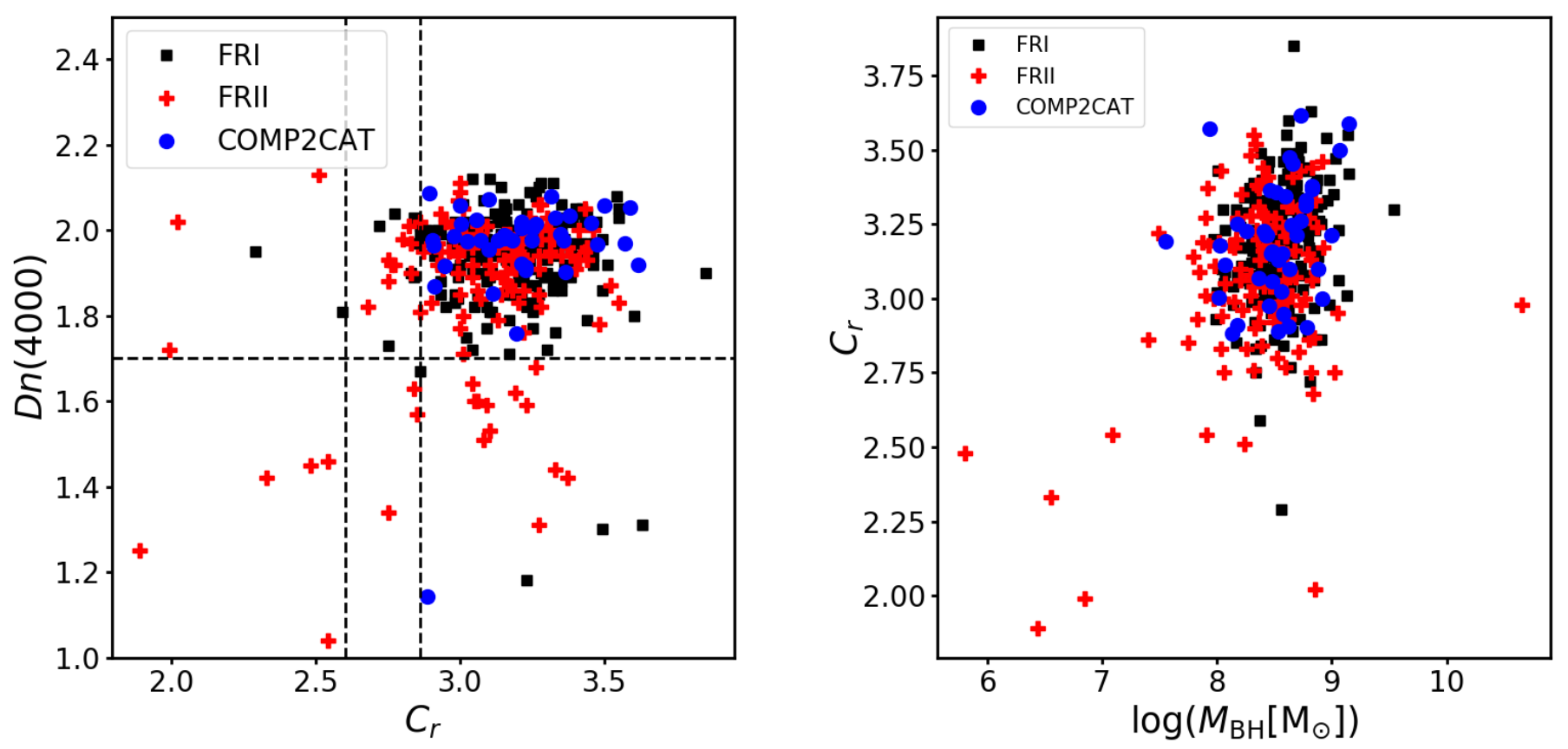

Fig. 14. Left: $\mathrm{Dn}(4000)$ index vs. concentration index $C_{r}$ for COMP2CAT (blue dots), FRICAT (black squares), and FRIICAT (red crosses). The black dashed lines represent the values of $C_{r}$ and $\mathrm{Dn}(4000)$ indices that, according to Nakamura et al. (2003), Shen et al. (2003), Strateva et al. (2001), Kauffmann et al. (2003), Bell et al. (2003), and Capetti \& Raiteri (2015) correspond to ETGs. Right: concentration index $C_{r}$ vs. logarithm of the black hole mass (in solar units) with the same color-coding as the image on the left.
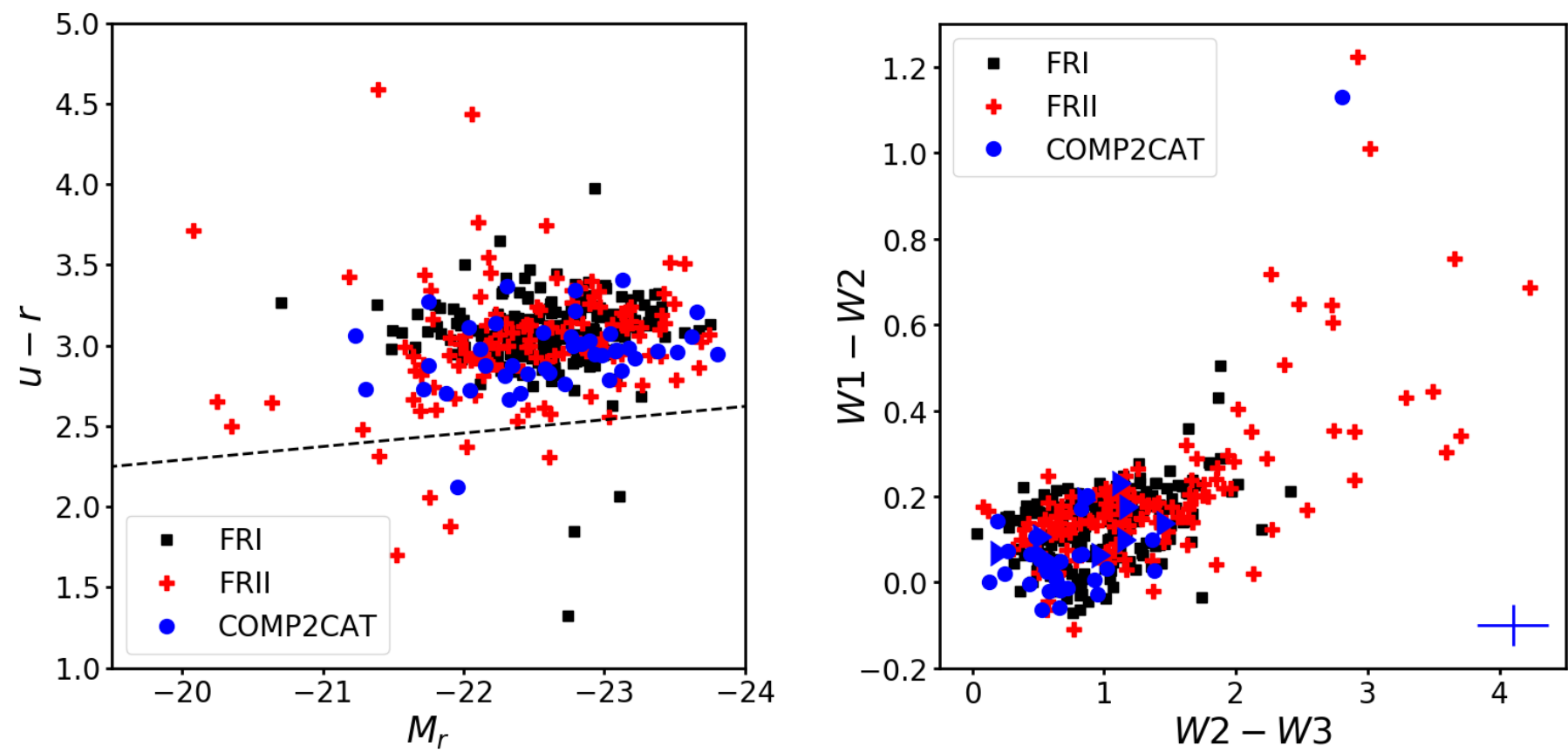

Fig. 15. Left: $u-r$ color vs. absolute $r$-band magnitude $\left(M_{r}\right)$ for COMP2CAT, FRICAT, and FRIICAT hosts (blue dots, black squares, and red crosses). The dashed line separates the blue ETG from the red sequence, following the definition published by Schawinski et al. (2009). Right: WISE mid-IR colors of COMP2CAT, FRICAT, and FRIICAT hosts with the same color-coding as in the Fig. 14. The blue cross in the bottom right corner of the plot represents the average error on the colors.

consistent with COMP2CAT sources being the low radio luminosity tail of FR-IIs and highlights the absence of HERGs in the tail. Having low radio luminosities and showing lower values of $E W_{\text {[OIII] }}$ than radio galaxies in FRIICAT, the line production could be mainly due to stellar processes rather than to the central AGN. This makes the $L_{[\mathrm{OIII}]}$ versus $L_{1.4}$ plane flatter towards the COMP2CAT region.
COMP2CAT sources lie in the lower part of the opticalradio luminosity plane (also known as the Ledlow-Owen plot; see Fig. 17). In particular, only $\sim 33 \%$ of COMP2CAT sources lie above the dashed line in Fig. 17, which corresponds to that reported in Ledlow \& Owen (1996) and marks the separation between the different FR classes of radio galaxies, while for FRIs and FRIIs the number of sources above this separation is $\sim 42 \%$ 


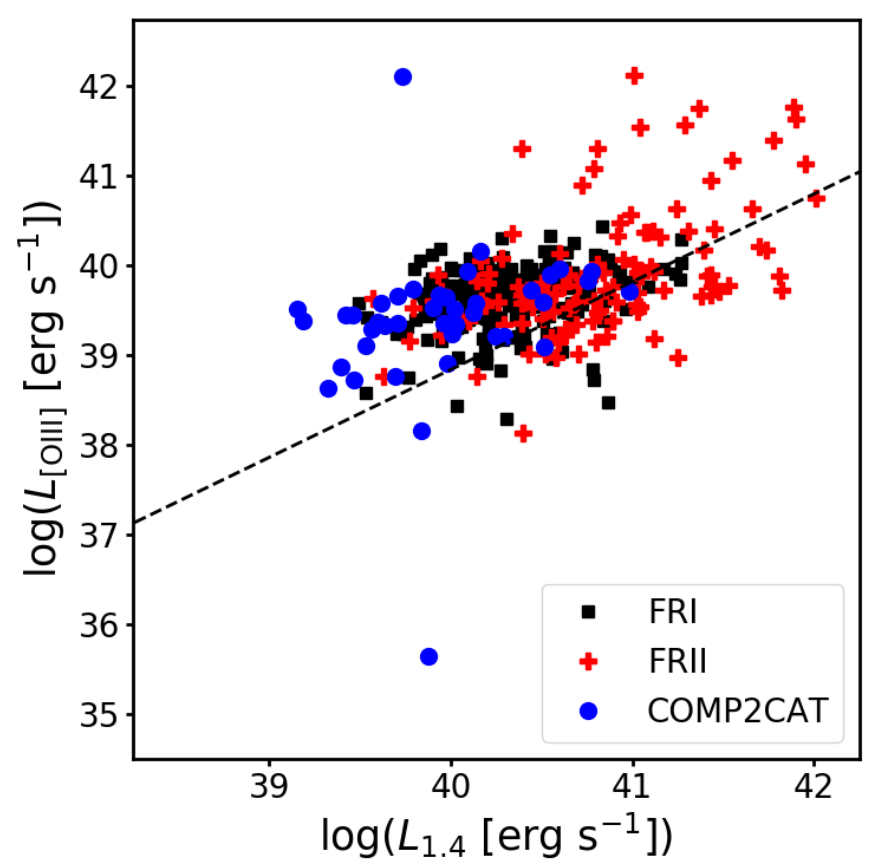

Fig. 16. [O III] line luminosity vs. radio luminosity at $1.4 \mathrm{GHz}$ for COMP2CAT sources (blue dots), FRICAT sources (black squares), and FRIICAT sources (red crosses). The black dashed line shows the linear correlation between these two quantities derived from the FR Is of the 3C sample from Buttiglione et al. (2010).

and $\sim 74 \%$, respectively. The higher fraction of COMP2CAT sources in the FRI region of the Ledlow-Owen plane is consistent with them being LERGs, like FRICAT sources, and in contrast with the FRIICAT population, which has a HERG fraction of $\sim 10 \%$.

Although COMP2CAT sources appear to be the low radio luminosity tail of FR IIs, the three populations (FRICAT, FRIICAT, and COMP2CAT sources) present the same ranges of $M_{B H}$, as shown in Fig. 18. Thus, the differences in the radio luminosity of these populations could arise from differences in their accretion rates and/or accretion mechanisms, COMP2CAT sources having less efficient accretion mechanisms than FR IIs. While COMP2CAT sources are almost exclusively LERGs, there are HERGs among the FR IIs in FRIICAT, so the hypothesis that the two populations have different accretion rates is consistent with HERGs having more efficient accretion mechanisms than LERGs, as proposed by several authors (Hardcastle et al. 2007; Balmaverde et al. 2008; Best \& Heckman 2012).

\section{Discussion and conclusions}

We built a catalog of 43 compact double sources selected from the Best \& Heckman (2012) sample restricted to the AGN with redshift $z<0.15$. Sources were selected if they fulfilled the two criteria: their radio emission does not extend beyond a $30 \mathrm{kpc}$ radius from the position of the optical host galaxy, and they show FRII-like morphologies, like the sources selected in FRIICAT (see Capetti et al. 2017a). This selection was carried out by visually inspecting the FIRST radio images of the sources. Only those identified as compact doubles by at least three out of five authors were included in the sample.

In order to improve the selection, we defined the asymmetric index $A$ (Eq. (1)) in such a way that very asymmetric sources (i.e., sources with one of the lobes much closer to the SDSS optical position than the other one) have $A \sim 1$, whereas sym-

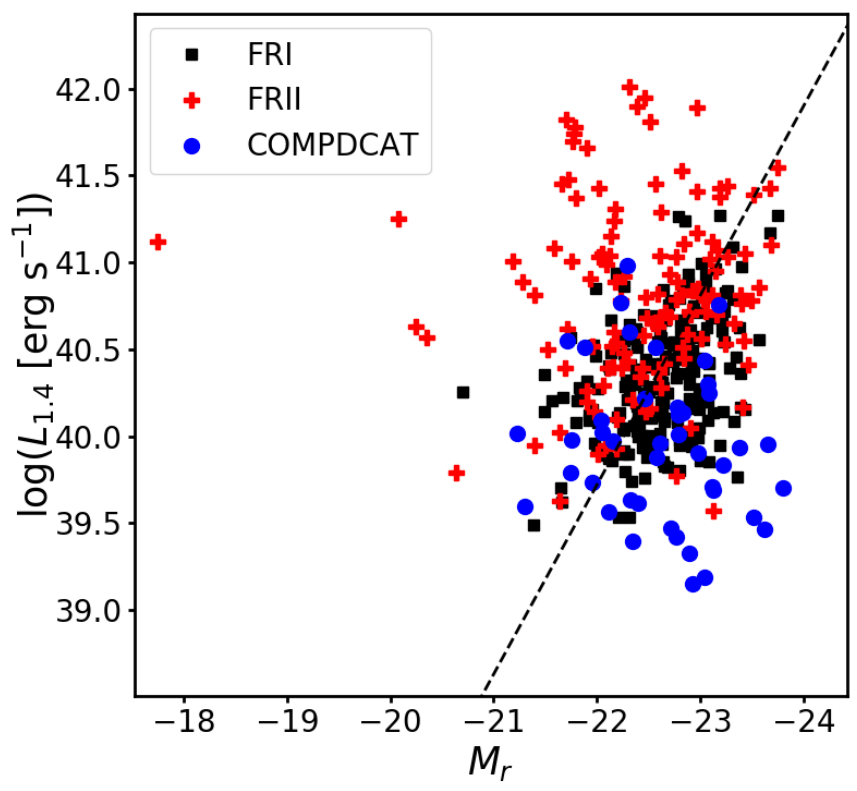

Fig. 17. NVSS radio luminosity vs. host absolute magnitude $\left(M_{r}\right)$ for COMP2CAT, FRICAT, and FRIICAT (blue dots, black squares, and red crosses, respectively). The dashed line shows the separation between FR I and FR II reported by Ledlow \& Owen (1996) to which we applied a correction of 0.34 mag to account for the different magnitude definition and the color transformation between the SDSS and Cousin systems.

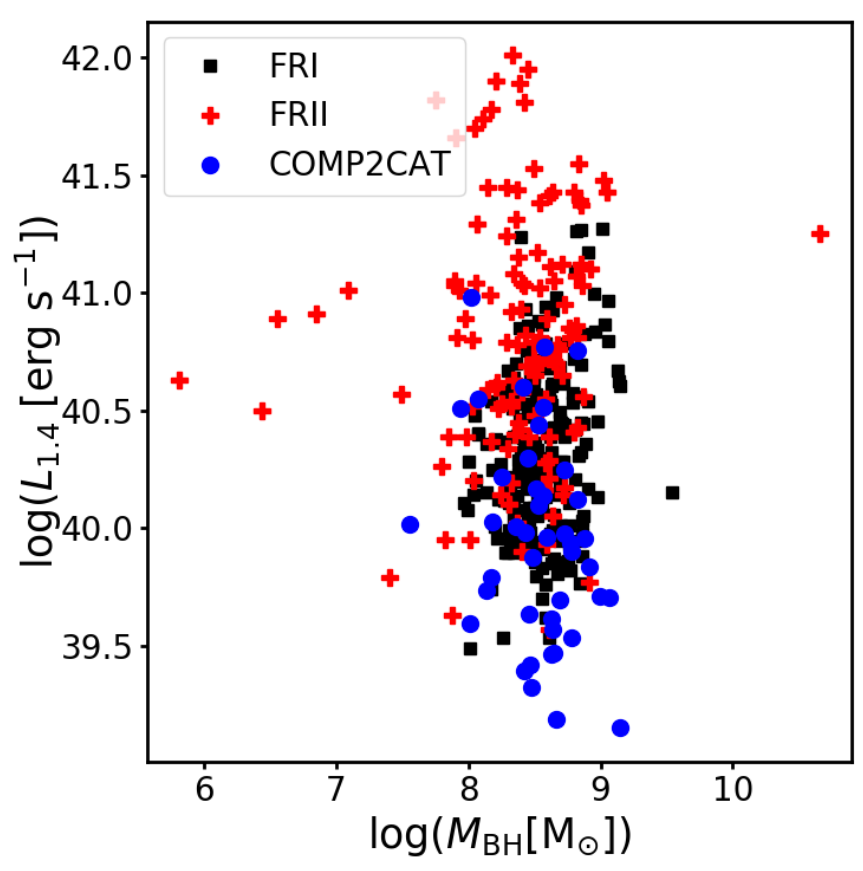

Fig. 18. Radio luminosity vs. black hole mass for COMP2CAT (blue dots), FRICAT (black squares), and FRIICAT (red crosses).

metric ones have $A \sim 0$. We excluded from our selection sources with $A \geq 0.5$.

Finally, we dropped from the selection sources with largescale NVSS and TGSS radio emission and with FRI morphologies revealed by high-resolution VLA radio maps. The resulting sample of 43 sources constitutes COMP2CAT. Since VLA radio maps are not available for all COMP2CAT sources, our sample may still be contaminated by sFRIs; it will be possible to deter- 
mine the degree of contamination with future observations from the VLA Sky Survey (VLASS ${ }^{8}$; Murphy \& Baum 2014).

Although our aim was to build a complete catalog of compact doubles, COMP2CAT is only $\sim 47 \%$ complete; $\sim 53 \%$ of sources were lost due to their low radio luminosities, their small projected linear sizes, and the incompleteness of the SDSS. However, we are not missing those with higher luminosities unless they have a projected linear size $\lesssim 10 \mathrm{kpc}$; so it remains that COMP2CAT sources are consistent with being the lower radio luminosity tail of both FRIs and FRIIs.

Given the estimated incompleteness of the catalog, we would expect to find $\sim 91$ sources up to $z=0.15$. This number is comparable with the number of FRI (219), FRII (122) and FR0 (108) sources found in this redshift range. Therefore, COMP2CAT sources constitute a significant fraction of the radio sources up to $z=0.15$.

All but one of the COMP2CAT sources are LERGs. This implies that either most compact doubles are LERGs or that HERGs mainly lie at $z>0.1$ where our catalog is the most incomplete. However, HERGs tend to have higher radio luminosities than LERGs, and therefore we would expect to find them if they existed at $0.1<z<0.15$. This lack of HERGs is consistent with COMP2CAT sources being the lower radio luminosity tail of the FRII sources.

Based on the purely morphological classification presented by Readhead (1995), COMP2CAT sources can be considered LSOs $(>15 \mathrm{kpc})$ and MSOs $(1-15 \mathrm{kpc})$. On the other hand, following the Orienti \& Dallacasa (2014) criteria, only one of the COMP2CAT sources can be considered a CSS source; therefore, we conclude that COMP2CAT sources constitute a different population from GPS/CSSs. This lack of GPS/CSSs in the sample could mean that we are missing those high radio luminosity sources with projected linear sizes smaller than $10 \mathrm{kpc}$ (CSOs/MSOs), which could be classified as CSSs.

The differences in the position of COMP2CAT sources with respect to FRIs and FRIIs in the $L_{[\mathrm{OIII}]}$ versus $L_{1.4}$ plane, in the $L_{1.4}$ versus $M_{\mathrm{BH}}$ plane, and in the Ledlow-Owen plot are consistent with COMP2CAT sources having lower radio luminosities than FR Is and FR IIs and with COMP2CAT sources being mostly LERGs (like the FRICAT sources), and in contrast with the FRIICAT sources (which have $~ 10 \%$ HERGs). Thus, these discrepancies between COMP2CAT sources and FR IIs could stem from differences in the accretion between LERGs and HERGs. The accretion of LERGs is indeed thought to be less efficient than that of HERGs. Were this hypothesis correct, COMP2CAT sources would be a population of radio galaxies with the lowest accretion rates.

An additional step to understand COMP2CAT sources would be to carry out a complete multi-frequency study of the catalog, including observations at low radio frequencies with the Low Frequency Array (LOFAR) and X-ray observations. Currently, only two COMP2CAT sources have been observed with Chandra (SDSS J081023.27+421625.8 and SDSS J113305.52+ 592013.7), both of them in galaxy clusters, identified using the 7 th and 4th SDSS releases by Yang et al. (2005, 2007) and Koester et al. (2007), respectively. Another two sources have been observed with XMM-Newton (SDSS J095341.37+014202.3 and SDSS J103801.77+414625.8), also in galaxy clusters identified using the SDSS by Shen et al. (2008) and Tempel et al. (2012). Finally, one more source has been observed with Swift (SDSS J101944.27-003817.8). This source is also part of a

\footnotetext{
8 Available at: https://science.nrao.edu/science/surveys/ vlass/vlass
}

galaxy cluster and was identified using the SDSS by Tempel et al. (2012). More Chandra observations are needed in order to characterize these sources completely. On the other hand, LOFAR and VLASS observations would allow us to characterize source radio spectra and to study their morphology at higher resolution in order to quantify the degree of contamination of our sample by sFRIs.

Finally, this catalog can be used in the future to better understand the role of compact double sources in the general evolutionary scheme of radio sources. In particular, by using LOFAR data we could compare our catalog to those selected at low radio frequencies, as was recently done by Hardcastle et al. (2019). Their sample lists 23244 radio-loud AGN, with $150 \mathrm{MHz}$ luminosities ranging from $10^{36}$ to $10^{45} \mathrm{erg} \mathrm{s}^{-1}$ and projected linear sizes between $1 \mathrm{pc}$ and $1 \mathrm{Mpc}$, obtained from the LOFAR Two Metre Sky Survey (LoTSS).

Acknowledgements. We are grateful to the anonymous referee for the constructive and valuable comments that improved the presentation of our manuscript. A.J. acknowledges the financial support (MASF_CONTR_FIN_18_01) from the Italian National Institute of Astrophysics under the agreement with the Instituto de Astrofisica de Canarias for the "Becas Internacionales para Licenciados y/o Graduados Convocatoria de 2017". This work is supported by the "Departments of Excellence 2018-2022" Grant awarded by the Italian Ministry of Education, University and Research (MIUR) (L. 232/2016). This research has made use of resources provided by the Compagnia di San Paolo for the grant awarded on the BLENV project (S1618_L1_MASF_01) and by the Ministry of Education, Universities and Research for the grant MASF_FFABR_17_01. F.M. acknowledges the financial contribution from the agreement ASI-INAF n.201714-H.0. A.P. acknowledges financial support from the Consorzio Interuniversitario per la Fisica Spaziale (CIFS) under the agreement related to the grant MASF_CONTR_FIN_18_02. L.O. acknowledges partial support from the INFN Grant InDark. A.S. was supported by the NASA contract contract NAS8-03060 (Chandra X-ray Center).

\section{References}

Abazajian, K. N., Adelman-McCarthy, J. K., Agüeros, M. A., et al. 2009, ApJS, 182,543

Baldi, R. D., \& Capetti, A. 2010, A\&A, 519, A48

Baldi, R. D., Capetti, A., \& Giovannini, G. 2015, A\&A, 576, A38

Baldi, R. D., Capetti, A., \& Massaro, F. 2018, A\&A, 609, A1

Balmaverde, B., Baldi, R. D., \& Capetti, A. 2008, A\&A, 486, 119

Balogh, M. L., Morris, S. L., Yee, H. K. C., Carlberg, R. G., \& Ellingson, E. 1999, ApJ, 527, 54

Becker, R. H., White, R. L., \& Helfand, D. J. 1995, ApJ, 450, 559

Bell, E. F., McIntosh, D. H., Katz, N., \& Weinberg, M. D. 2003, ApJS, 149, 289

Bennett, C. L., Larson, D., Weiland, J. L., \& Hinshaw, G. 2014, ApJ, 794, 135

Best, P. N. 2009, Astron. Nachr., 330, 184

Best, P. N., \& Heckman, T. M. 2012, MNRAS, 421, 1569

Brinchmann, J., Charlot, S., White, S. D. M., et al. 2004, MNRAS, 351, 1151

Bodo, G., Mamatsashvili, G., Rossi, P., \& Mignone, A. 2013, MNRAS, 434, 3030

Buttiglione, S., Capetti, A., Celotti, A., et al. 2010, A\&A, 509, A6

Capetti, A., \& Baldi, R. D. 2011, A\&A, 529, A126

Capetti, A., \& Raiteri, C. M. 2015, A\&A, 580, A73

Capetti, A., Massaro, F., \& Baldi, R. D. 2017a, A\&A, 601, A81

Capetti, A., Massaro, F., \& Baldi, R. D. 2017b, A\&A, 598, A49

Condon, J. J., Cotton, W. D., Greisen, E. W., et al. 1998, AJ, 115, 1693

D'Abrusco, R., Massaro, F., Paggi, A., et al. 2014, ApJS, 215, 14

Fanaroff, B. L., \& Riley, J. M. 1974, MNRAS, 167, 31P

Fanti, C., Fanti, R., Dallacasa, D., et al. 1995, A\&A, 302, 317

Ghisellini, G. 2011, AIP Conf. Proc., 1381, 180

Hardcastle, M. J., Evans, D. A., \& Croston, J. H. 2007, MNRAS, 376, 1849

Hardcastle, M. J., Williams, W. L., Best, P. N., et al. 2019, A\&A, 622, A12

Kauffmann, G., Heckman, T. M., White, S. D. M., et al. 2003, MNRAS, 341, 33

Koester, B. P., McKay, T. A., Annis, J., et al. 2007, ApJ, 660, 239

Kunert-Bajraszewska, M., Gawroński, M. P., Labiano, A., \& Siemiginowska, A. 2010, MNRAS, 408, 2261

Labiano, A. 2006, PhD Thesis, Space Telescope Science Institute Kapteyn Astronomical Institute 
Labiano, A. 2009, Astron. Nachr., 330, 241

Ledlow, M. J., \& Owen, F. N. 1996, AJ, 112, 9

Lin, Y. T., Shen, Y., Strauss, M. A., Richards, G. T., \& Lunnan, R. 2010, ApJ, 723, 1119

Massaro, F., Masetti, N., D’Abrusco, R., Paggi, A., \& Funk, S. 2014, AJ, 148 66

Montero-Dorta, A. D., \& Prada, F. 2009, MNRAS, 399, 1106

Murphy, E. J., \& Baum, S. 2014, NRAO [White paper]

Nakamura, O., Fukugita, M., Yasuda, N., et al. 2003, AJ, 125, 1682

O’Dea, C. P. 1998, PASP, 110, 493

Orienti, M., \& Dallacasa, D. 2014, MNRAS, 438, 463

Owen, F. N., \& Rudnick, L. 1976, ApJ, 205, L1

Phillips, R. B., \& Mutel, R. L. 1982, A\&A, 106, 21

Readhead, A. C. S. 1995, Proc. Natl. Acad. Sci., 92, 11447

Schawinski, K., Lintott, C., Thomas, D., et al. 2009, MNRAS, 396, 818

Schoenmakers, A. P., Bruyn, A. G., Röttgering, H. J. A., et al. 2000, MNRAS, 315,371

Shen, S., Mo, H. J., White, S. D. M., et al. 2003, MNRAS, 343, 978
Shen, S., Kauffmann, G., von der Linden, A., White, S. D. M., \& Best, P. N. 2008, MNRAS, 389, 1074

Snellen, I. A. G., Mack, K.-H., Schilizzi, R. T., \& Tschager, W. 2004, MNRAS, 348,227

Sobolewska, M., Siemiginowska, A., Guainazzi, M., et al. 2019, ApJ, 871, 71

Strateva, I., Ivezić, Ž., Knapp, G. R., et al. 2001, AJ, 122, 1861

Strauss, M. A., Weinberg, D. H., Lupton, R. H., et al. 2002, AJ, 124, 1810

Tempel, E., Tago, E., \& Liivamägi, L. J. 2012, A\&A, 540, A106

Tremaine, S., Gebhardt, K., Bender, R., et al. 2002, ApJ, 574, 740

Tremonti, C. A., Heckman, T. M., Kauffmann, G., et al. 2004, ApJ, 613, 898

Wing, J. D., \& Blanton, E. L. 2011, AJ, 141, 3

Wright, E. L., Eisenhardt, P. R. M., Mainzer, A. K., et al. 2010, AJ, 140, 1868

Yang, X., Mo, H. J., van den Bosch, F. C., \& Jing, Y. P. 2005, MNRAS, 356, 1293

Yang, X., Mo, H. J., van den Bosch, F. C., et al. 2007, ApJ, 671, 153 
Appendix A: Excluded sources based on their VLA emission
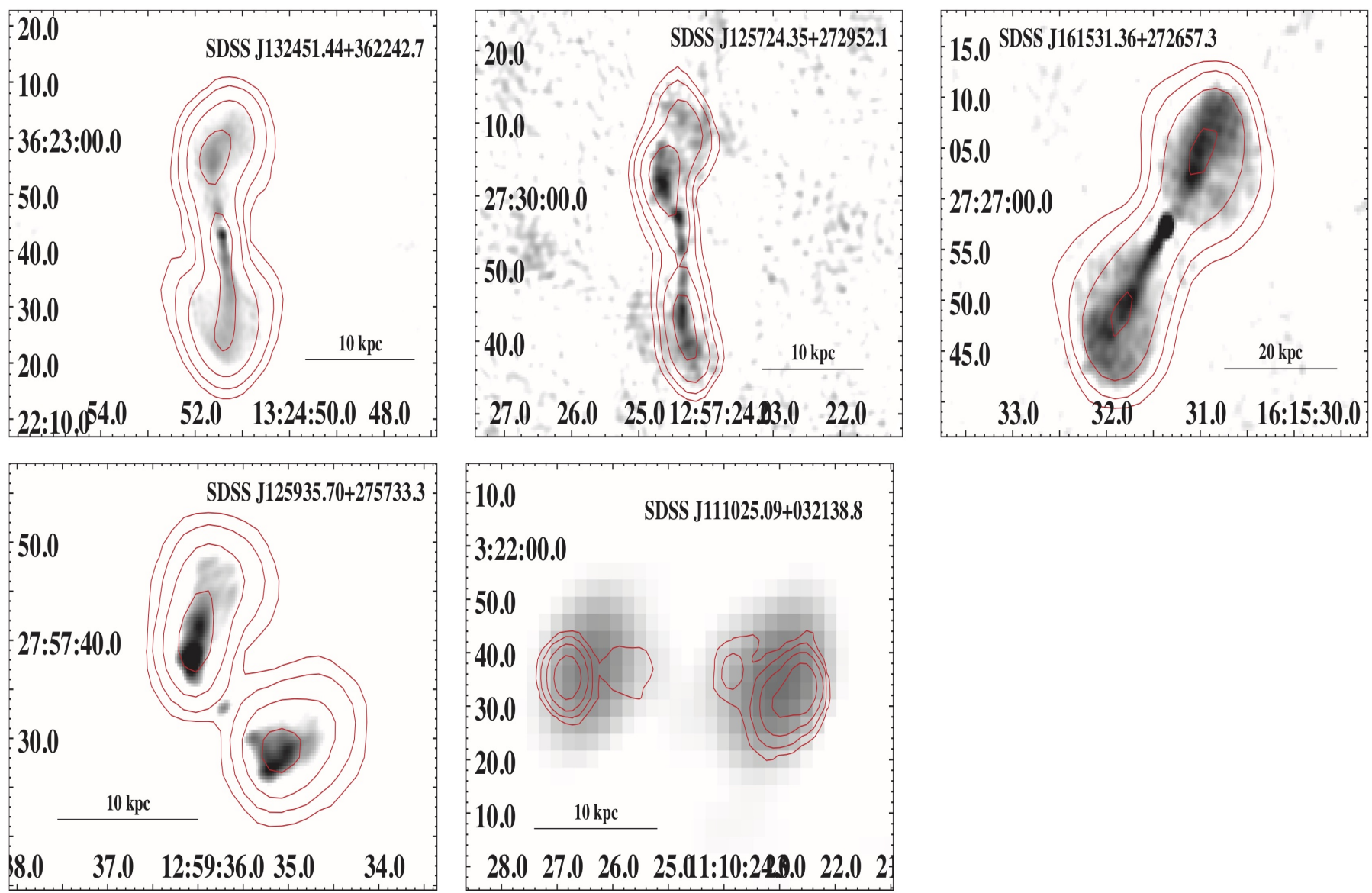

Fig. A.1. Images of sources with different radio morphologies seen in VLA. Contours are drawn using the parameters shown in Table E.1. The first three images correspond to small FRI sources like those selected in Capetti et al. (2017b) (the first was excluded from our sample because of its FIRST morphology). The fourth image corresponds to a WAT source (see Owen \& Rudnick 1976) and the last image shows a star-forming galaxy excluded from the sample. 
Appendix B: Sources with extended emisson in NVSS and TGSS
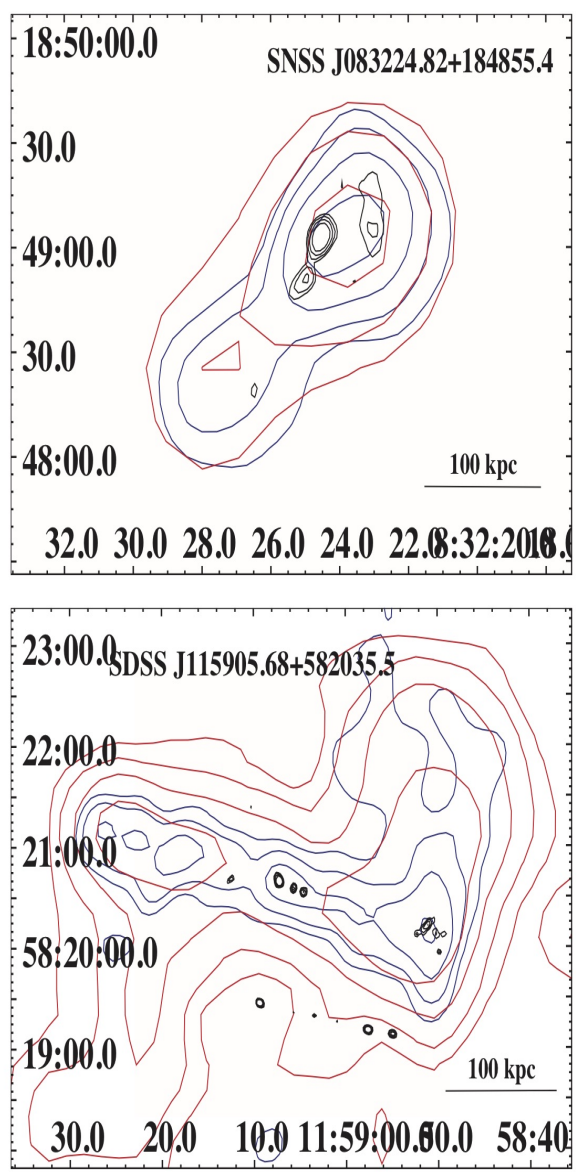
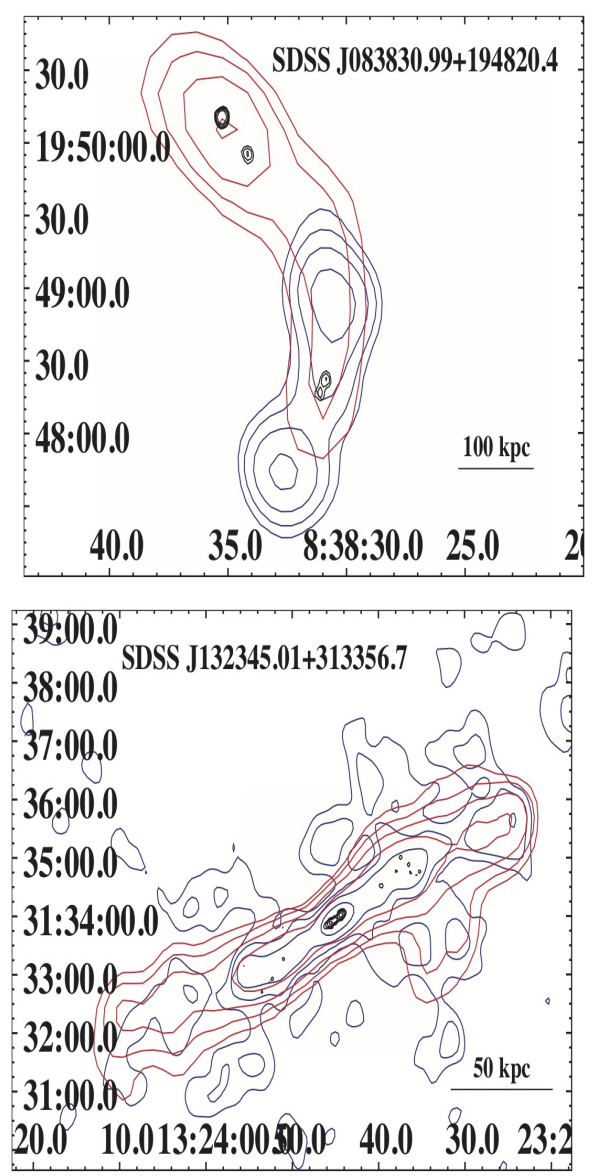
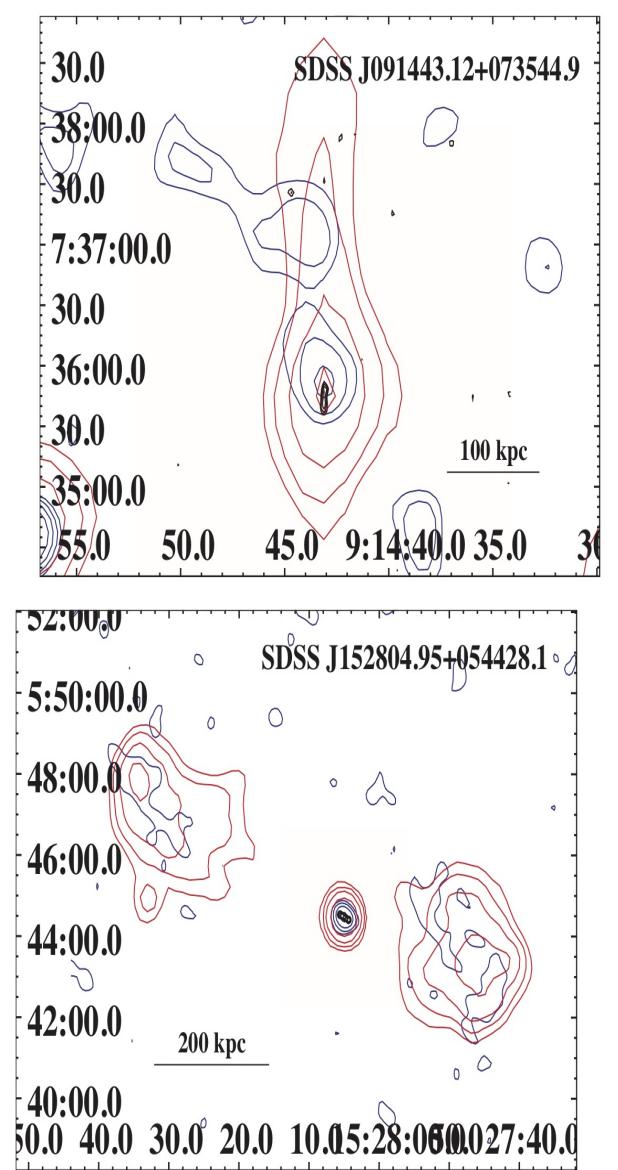

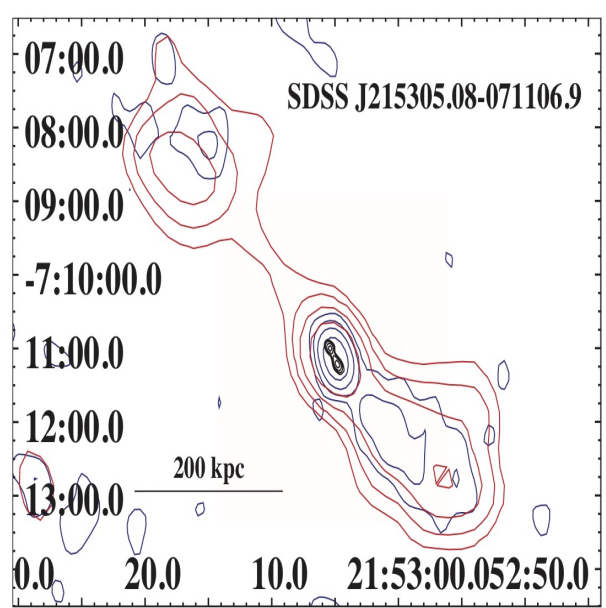

Fig. B.1. Images of sources with extended emission detected in NVSS. Black, red, and blue contours correspond to the emission seen in FIRST, NVSS, and TGSS. Contours are drawn using the parameters shown in Table E.2. 


\section{Appendix C: Porperties of COMP2CAT sources}

Table C.1. Properties of COMP2CAT sources.

\begin{tabular}{|c|c|c|c|c|c|c|c|c|c|c|c|c|c|}
\hline SDSS name & $z$ & $S_{1.4}$ & $S_{\text {[OIII] }}$ & $M_{r}$ & $\operatorname{Dn}(4000)$ & $\sigma_{*}$ & $C_{r}$ & $L_{1.4}$ & $L_{[\mathrm{OIII}]}$ & $M_{\mathrm{BH}}$ & $L S$ & $A$ & $\alpha$ \\
\hline J073600.87+273926.0 & 0.079 & 12.0 & 18.2 & -22.766 & 1.99 & 3.15 & 243 & 39.42 & 39.46 & 0.5 & 14.6 & 0.34 & 0.70 \\
\hline J074132.98+475215.6 & 0.127 & 12.4 & 0.0 & -22.579 & 2.02 & 3.06 & 245 & 39.88 & 35.64 & 8.5 & 29.2 & 0.06 & \\
\hline J074641.45+184405.4 & 0.051 & 17.4 & 38.4 & -23.037 & 2.02 & 3.45 & 271 & 39.19 & 39.39 & 8.7 & 12.1 & 0.21 & 0.66 \\
\hline J081023.27+421625.8 & 0.064 & 10.3 & 33.0 & -22.93 & 2.05 & 3.59 & 358 & 39.15 & 39.51 & 9.1 & 14.3 & 0.13 & \\
\hline J081104.30+355908.3 & 0.082 & 58.6 & 23.0 & -22.835 & 1.98 & 3.02 & 256 & 40.14 & 39.58 & 8.6 & 11.8 & 0.03 & \\
\hline J082033.79+395142.4 & 0.102 & 25.4 & 3.0 & -21.751 & 2.02 & 3.21 & 238 & 39.98 & 38.91 & 8.4 & 30.7 & 0.05 & 0.53 \\
\hline J083053.58+231035.7 & 0.145 & 13.0 & 5.7 & -21.231 & 1.76 & 3.19 & 144 & 40.02 & 39.51 & 7.6 & 25.8 & 0.47 & 0.67 \\
\hline J084517.83+303027.4 & 0.106 & 6.0 & 2.5 & -22.342 & 1.92 & 3.21 & 237 & 39.39 & 38.87 & 8.4 & 44.2 & 0.16 & \\
\hline J090311.14+540351.6 & 0.083 & 73.0 & 9.5 & -23.084 & 2.01 & 3.26 & 281 & 40.25 & 39.22 & 8.7 & 11.8 & 0.01 & 0.68 \\
\hline J091134.75+125538.1 & 0.05 & 394.6 & 67.1 & -21.878 & 1.97 & 3.57 & 179 & 40.51 & 39.6 & 7.9 & 40.0 & 0.02 & 0.69 \\
\hline J095341.37+014202.3 & 0.098 & 9.8 & 5.1 & -23.519 & 1.98 & 2.9 & 290 & 39.53 & 39.1 & 8.8 & 40.3 & 0.26 & 1.21 \\
\hline $\mathrm{J} 100622.41+301332.9$ & 0.114 & 30.7 & 42.7 & -22.775 & 1.98 & 3.36 & 249 & 40.17 & 40.16 & 8.5 & 17.1 & 0.06 & 0.36 \\
\hline $\mathrm{J} 101653.82+002857.0$ & 0.116 & 10.9 & 3684.6 & -21.957 & 1.14 & 2.88 & 201 & 39.73 & 42.12 & 8.1 & 34.0 & 0.28 & 0.53 \\
\hline $\mathrm{J} 101944.27-003817.8$ & 0.094 & 9.3 & 12.7 & -23.621 & 1.96 & 2.91 & 266 & 39.46 & 39.45 & 8.6 & 16.5 & 0.2 & 0.57 \\
\hline $\mathrm{J} 103801.77+414625.8$ & 0.125 & 22.9 & 7.2 & -22.791 & 0.0 & 3.37 & 298 & 40.12 & 39.47 & 8.8 & 22.3 & 0.16 & 0.76 \\
\hline $\mathrm{J} 103842.52+120315.6$ & 0.092 & 31.5 & 20.9 & -22.156 & 1.92 & 3.61 & 282 & 39.98 & 39.65 & 8.7 & 18.9 & 0.02 & 0.51 \\
\hline $\mathrm{J} 104254.02+282559.0$ & 0.055 & 40.4 & 52.0 & -22.402 & 1.97 & 3.47 & 266 & 39.62 & 39.58 & 8.6 & 27.4 & 0.21 & 0.46 \\
\hline $\mathrm{J} 111109.58+393552.0$ & 0.078 & 40.4 & 31.0 & -23.378 & 2.03 & 3.33 & 289 & 39.94 & 39.68 & 8.8 & 8.5 & 0.07 & 0.56 \\
\hline J113305.52+592013.7 & 0.133 & 13.6 & -3.5 & -23.655 & 1.96 & 3.1 & 307 & 39.96 & & 8.9 & 17.1 & 0.14 & 0.91 \\
\hline $\mathrm{J} 113643.49+545446.8$ & 0.055 & 41.8 & 29.1 & -22.321 & 1.9 & 3.37 & 242 & 39.64 & 39.33 & 8.5 & 10.0 & 0.02 & 0.55 \\
\hline J115050.98-031113.0 & 0.129 & 52.6 & 2.8 & -22.567 & 1.98 & 3.15 & 257 & 40.51 & 39.09 & 8.6 & 20.8 & 0.09 & 0.48 \\
\hline $\mathrm{J} 122208.81+073329.6$ & 0.137 & 14.3 & 3.4 & -22.788 & 1.98 & 3.07 & 229 & 40.01 & 39.24 & 8.4 & 25.7 & 0.23 & 0.81 \\
\hline $\mathrm{J} 125319.21+475335.2$ & 0.139 & 16.9 & 16.4 & -22.037 & 1.98 & 3.13 & 252 & 40.09 & 39.93 & 8.5 & 16.1 & 0.04 & 0.39 \\
\hline $\mathrm{J} 130107.54-032652.5$ & 0.083 & 112.6 & 30.1 & -23.041 & 2.09 & 2.89 & 252 & 40.44 & 39.72 & 8.5 & 16.9 & 0.07 & 0.45 \\
\hline $\mathrm{J} 131705.93+435713.2$ & 0.052 & 43.7 & 36.6 & -21.305 & 2.02 & 3.0 & 187 & 39.59 & 39.37 & 8.0 & 23.4 & 0.19 & 0.5 \\
\hline $\mathrm{J} 131945.31+603043.0$ & 0.07 & 208.4 & 65.9 & -21.714 & 1.85 & 3.11 & 194 & 40.55 & 39.9 & 8.1 & 26.7 & 0.17 & 0.75 \\
\hline $\mathrm{J} 132031.47-012718.5$ & 0.083 & 15.4 & 11.5 & -22.119 & 2.07 & 3.1 & 267 & 39.57 & 39.29 & 8.6 & 32.5 & 0.04 & \\
\hline J132602.39+364759.3 & 0.054 & 957.0 & 71.5 & -22.292 & 1.98 & 3.18 & 188 & 40.98 & 39.71 & 8.0 & 21.4 & 0.11 & 0.62 \\
\hline $\mathrm{J} 132649.30+164948.0$ & 0.08 & 41.0 & 14.5 & -22.61 & 1.99 & 3.35 & 261 & 39.96 & 39.36 & 8.6 & 25.2 & 0.01 & 0.37 \\
\hline J133917.34-015048.7 & 0.089 & 58.2 & -0.0 & -22.456 & 1.91 & 3.23 & 215 & 40.22 & & 8.3 & 12.8 & 0.34 & 0.45 \\
\hline $\mathrm{J} 135338.43+360802.4$ & 0.027 & 128.9 & 32.6 & -22.716 & 1.98 & 3.25 & 269 & 39.47 & 38.72 & 8.7 & 39.0 & 0.07 & 0.5 \\
\hline $\mathrm{J} 135347.34+515734.3$ & 0.132 & 90.4 & 18.7 & -22.225 & 1.92 & 2.95 & 258 & 40.77 & 39.94 & 8.6 & 17.0 & 0.2 & 0.62 \\
\hline $\mathrm{J} 144647.43+032527.1$ & 0.125 & 18.3 & 5.4 & -22.047 & 1.87 & 2.91 & 206 & 40.03 & 39.35 & 8.2 & 12.2 & 0.23 & \\
\hline $\mathrm{J} 144731.24+330606.2$ & 0.088 & 73.0 & 8.4 & -23.076 & 1.99 & 2.98 & 240 & 40.3 & 39.21 & 8.4 & 10.7 & 0.2 & 0.52 \\
\hline $\mathrm{J} 145604.88+472712.4$ & 0.087 & 211.4 & 35.9 & -23.175 & 2.03 & 3.38 & 298 & 40.75 & 39.84 & 8.8 & 23.8 & 0.04 & 0.61 \\
\hline $\mathrm{J} 145858.83+130145.9$ & 0.112 & 10.7 & 1.8 & -23.13 & 2.01 & 3.21 & 276 & 39.69 & 38.76 & 8.7 & 18.9 & 0.17 & \\
\hline $\mathrm{J} 151135.87+191228.0$ & 0.08 & 22.6 & 29.0 & -23.805 & 2.06 & 3.5 & 342 & 39.71 & 39.67 & 9.1 & 22.1 & 0.16 & 0.74 \\
\hline J155749.61+161836.6 & 0.037 & 113.4 & 70.4 & -23.118 & 2.0 & 3.21 & 328 & 39.71 & 39.35 & 9.0 & 19.4 & 0.19 & 0.43 \\
\hline J160818.19+374335.3 & 0.102 & 5.6 & 1.6 & -22.894 & 1.99 & 3.16 & 244 & 39.32 & 38.63 & 8.5 & 15.2 & 0.06 & \\
\hline $\mathrm{J} 162401.10+204018.4$ & 0.1 & 17.2 & 21.2 & -21.749 & 2.01 & 3.25 & 205 & 39.79 & 39.74 & 8.2 & 21.1 & 0.26 & 0.33 \\
\hline $\mathrm{J} 164452.86+341251.3$ & 0.085 & 31.2 & 18.6 & -22.982 & 2.08 & 3.31 & 290 & 39.9 & 39.53 & 8.8 & 14.8 & 0.1 & 0.54 \\
\hline $\mathrm{J} 165644.31+324321.8$ & 0.147 & 48.4 & 15.9 & -22.309 & 1.91 & 3.23 & 235 & 40.6 & 39.97 & 8.4 & 20.1 & 0.07 & 0.45 \\
\hline $\mathrm{J} 171659.25+321445.0$ & 0.111 & 15.3 & 0.5 & -23.219 & 2.06 & 3.0 & 313 & 39.83 & 38.16 & 8.9 & 23.4 & 0.23 & 0.64 \\
\hline
\end{tabular}

Notes. Column description: (1) source name; (2) redshift; (3) NVSS $1.4 \mathrm{GHz}$ flux density [mJy]; (4) [O III] flux density [10 $\left.0^{-17} \mathrm{erg} \mathrm{cm}^{-2} \mathrm{~s}^{-1}\right]$; (5) SDSS DR7 $r$-band $A B$ magnitude; (6) Dn(4000) index; (7) stellar velocity dispersion [km s${ }^{-1}$ ]; (8) concentration index; (9) logarithm of the radio luminosity [ $\mathrm{erg} \mathrm{s}^{-1}$ ]; (10) logarithm of the [O III] line luminosity [ $\left.\mathrm{erg} \mathrm{s}^{-1}\right]$; (11) logarithm of the black hole mass $\left[M_{\odot}\right]$; (12) radio linear size [kpc]; (13) asymmetric index; (14) spectral index between $1.4 \mathrm{GHz}$ and $150 \mathrm{MHz}$. 
Appendix D: FIRST, NVSS, and TGSS images of the 43 COMP2CAT sources
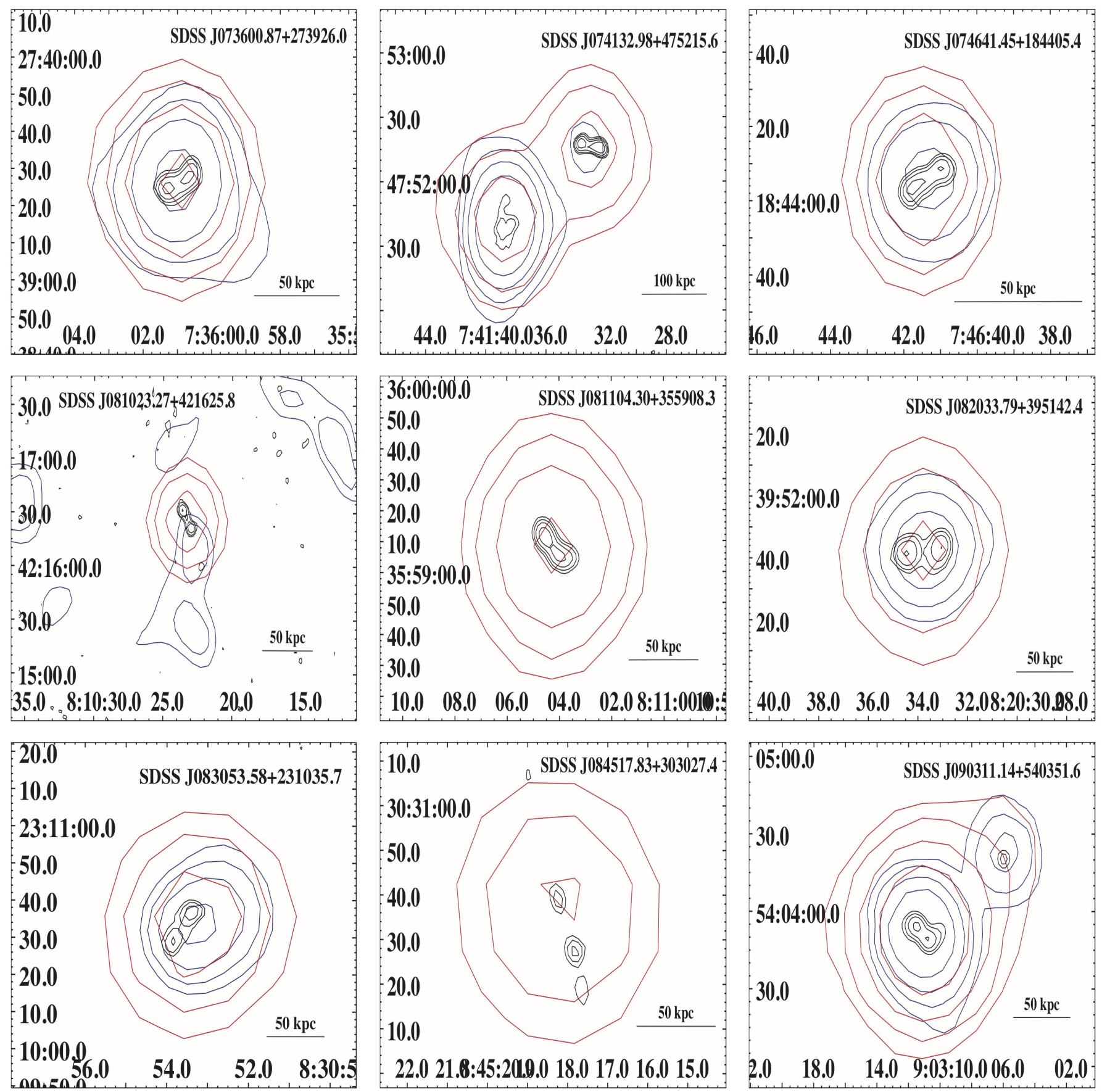

Fig. D.1. Images of COMP2CAT sources. Black, red, and blue contours correspond to the emission seen in FIRST, NVSS, and TGSS. Contours are drawn using the parameters shown in Table E.3. 
A. Jimenez-Gallardo et al.: COMP2CAT: hunting compact double radio sources
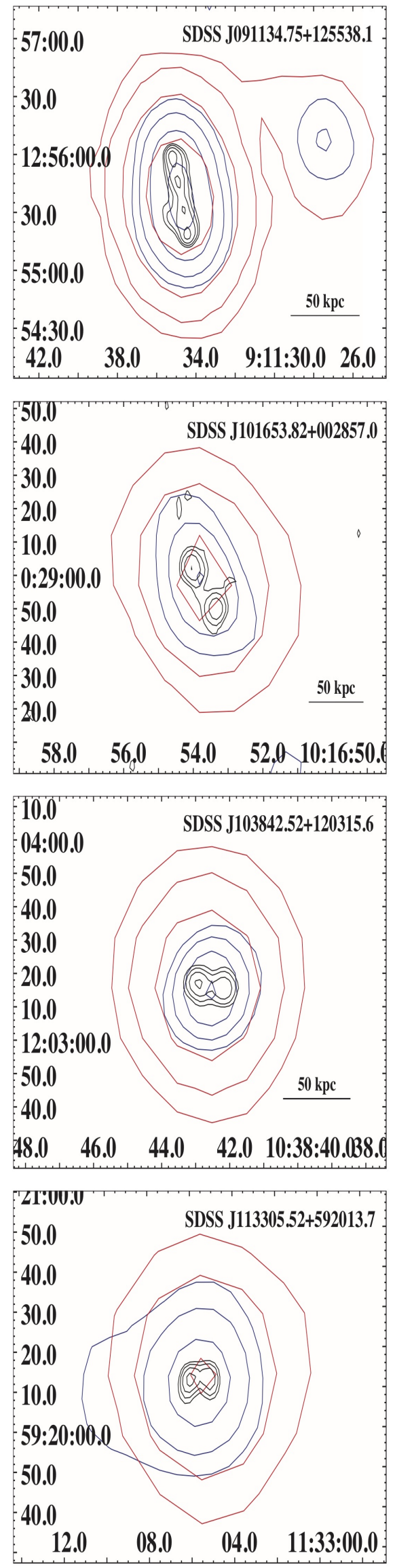
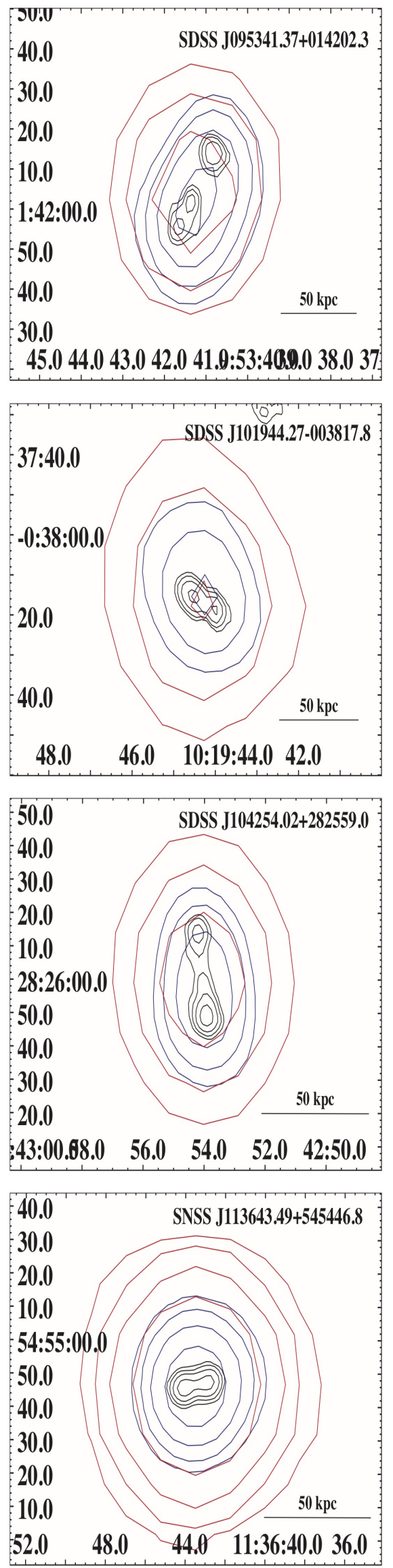

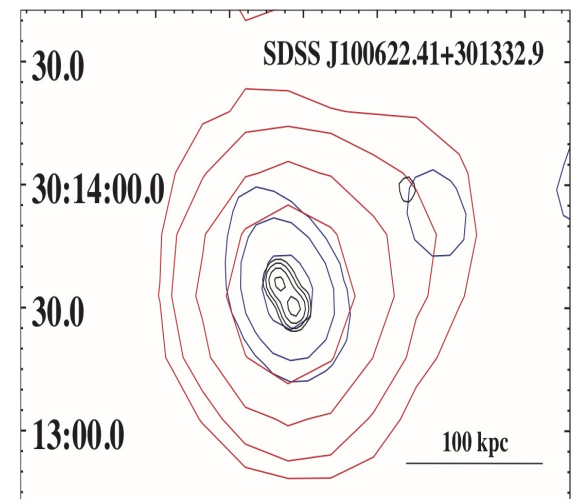

$28.0 \quad 26.0 \quad 24.0 \quad 22.010: 06: 201.0 .0 \quad 16.0$
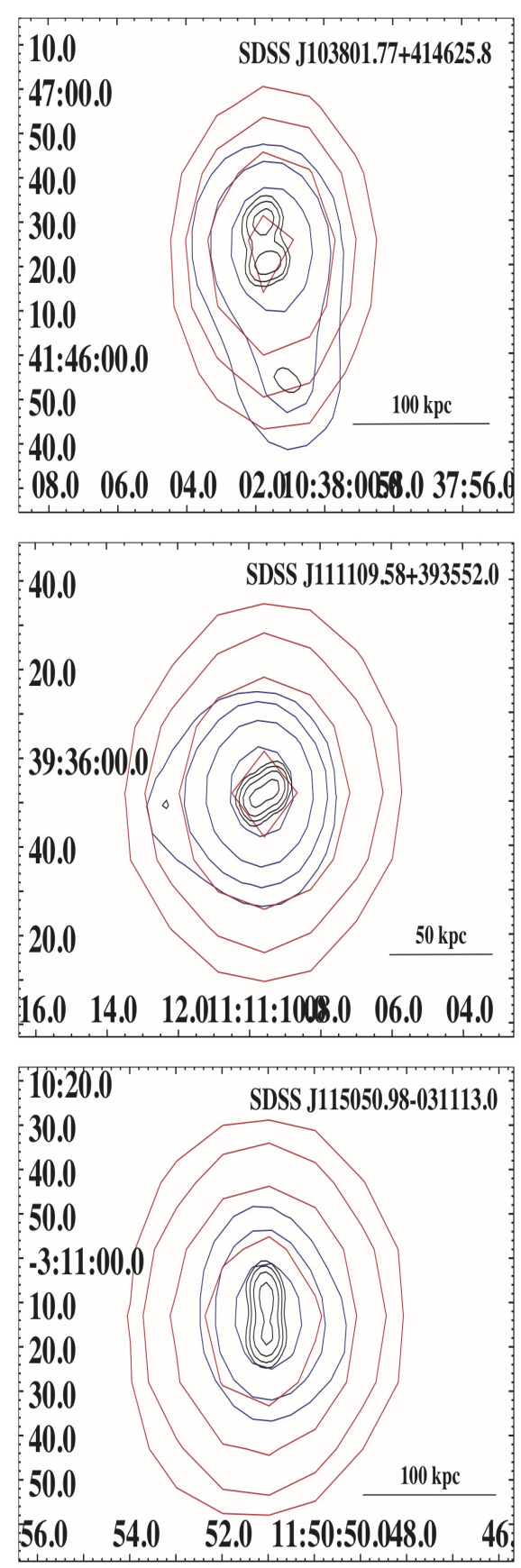

Fig. D.1. continued. 

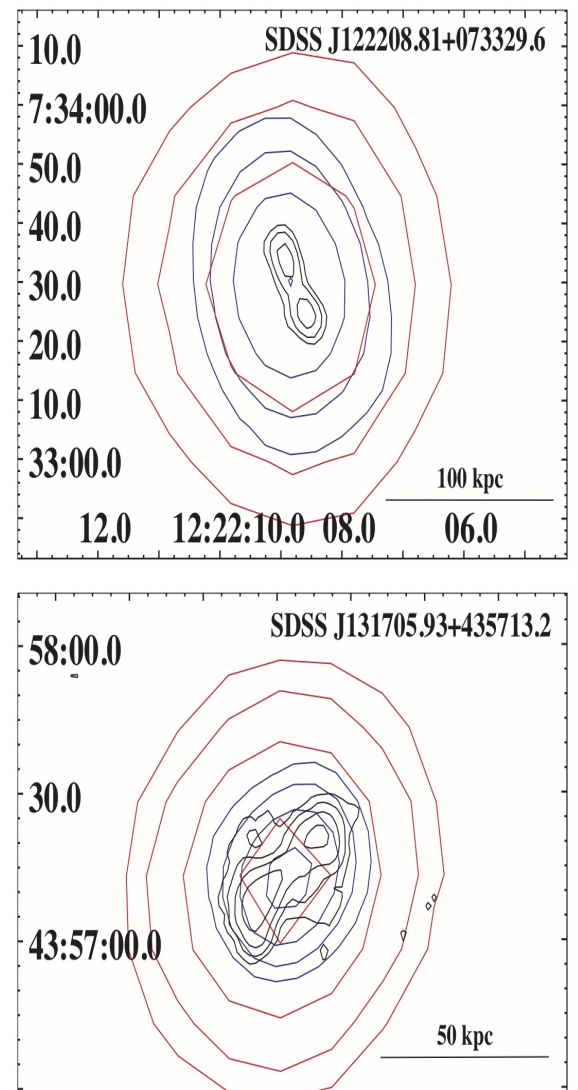

$\begin{array}{llllll}12.0 & 10.0 & 08.0 & 06.0 & 04.0 & 02.013: 17: 00\end{array}$
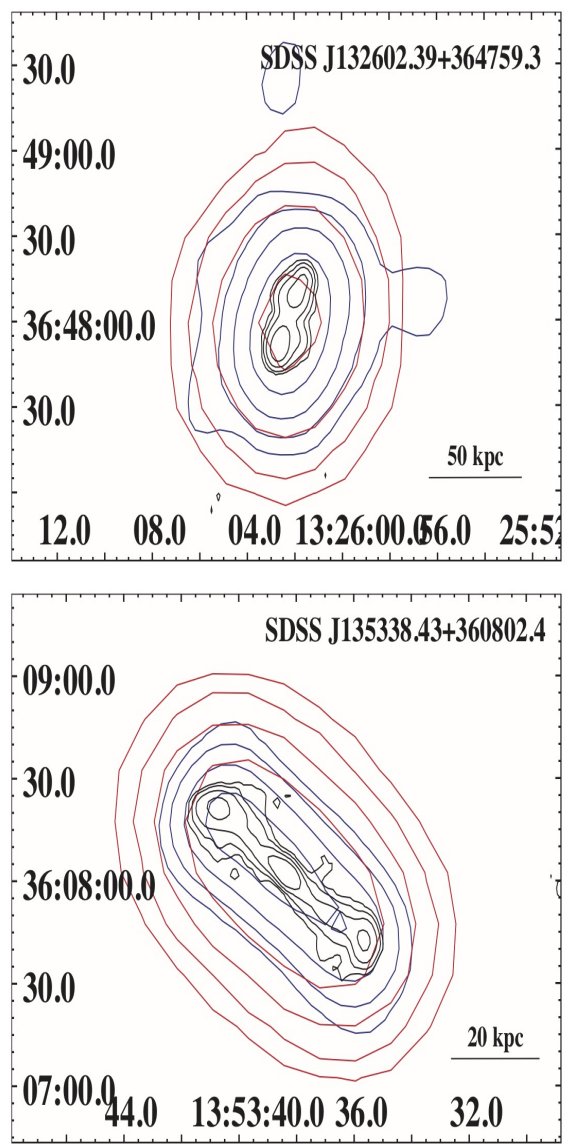

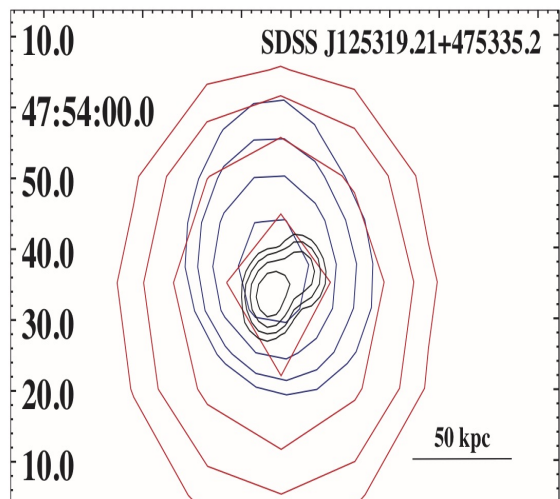

$\begin{array}{lllll}24.0 & 22.0 & 12: 53: 20.018 .0 & 16.0 & 14 .\end{array}$
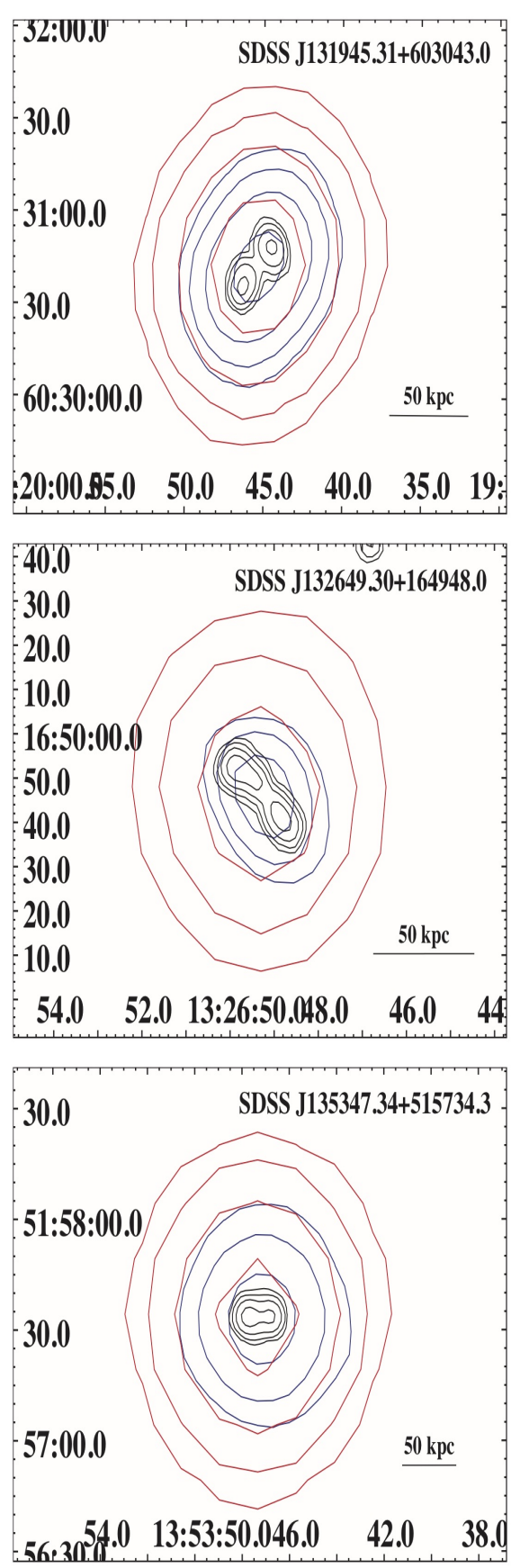

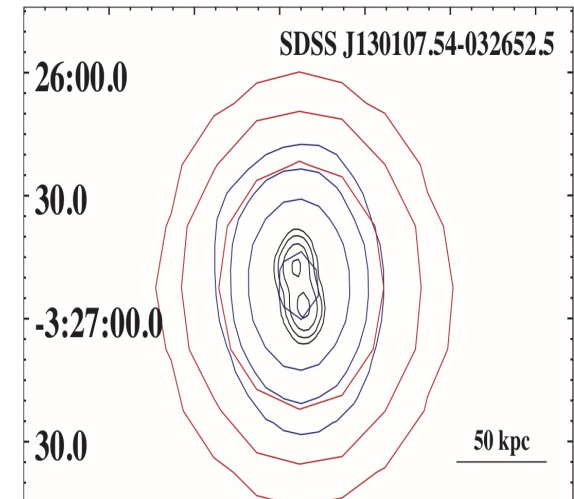

$\begin{array}{llllll}4.0 & 12.013: 01: 1008.0 & 06.0 & 04.0 & 02.0\end{array}$
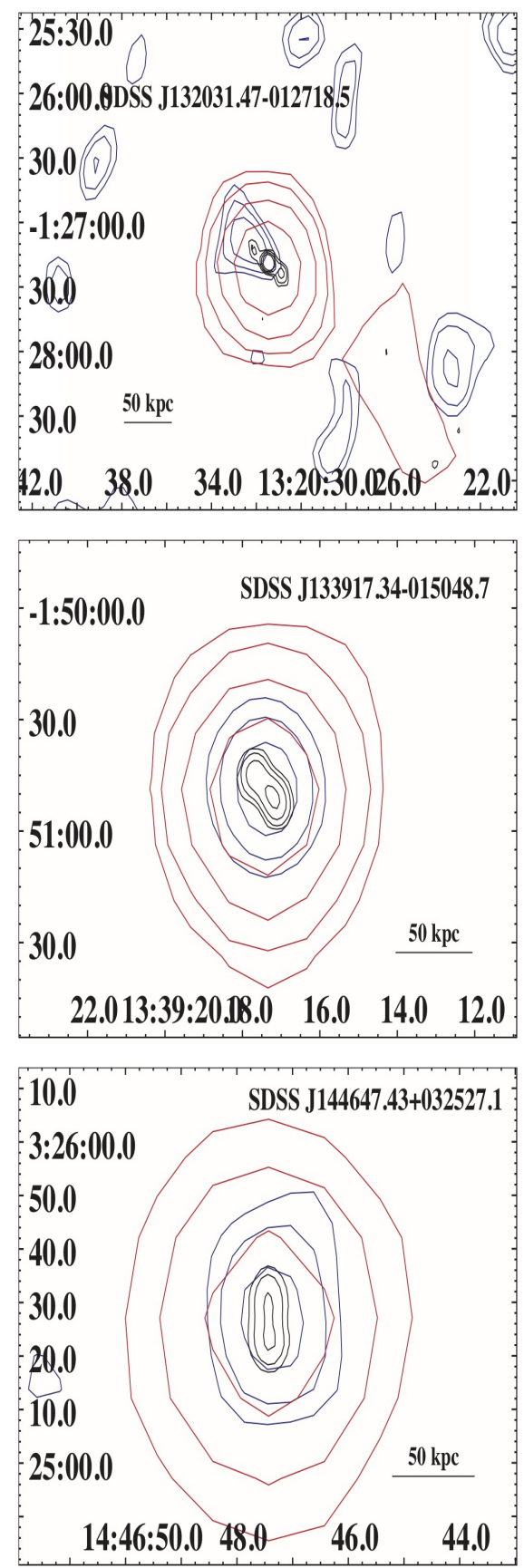

Fig. D.1. continued. 
A. Jimenez-Gallardo et al.: COMP2CAT: hunting compact double radio sources
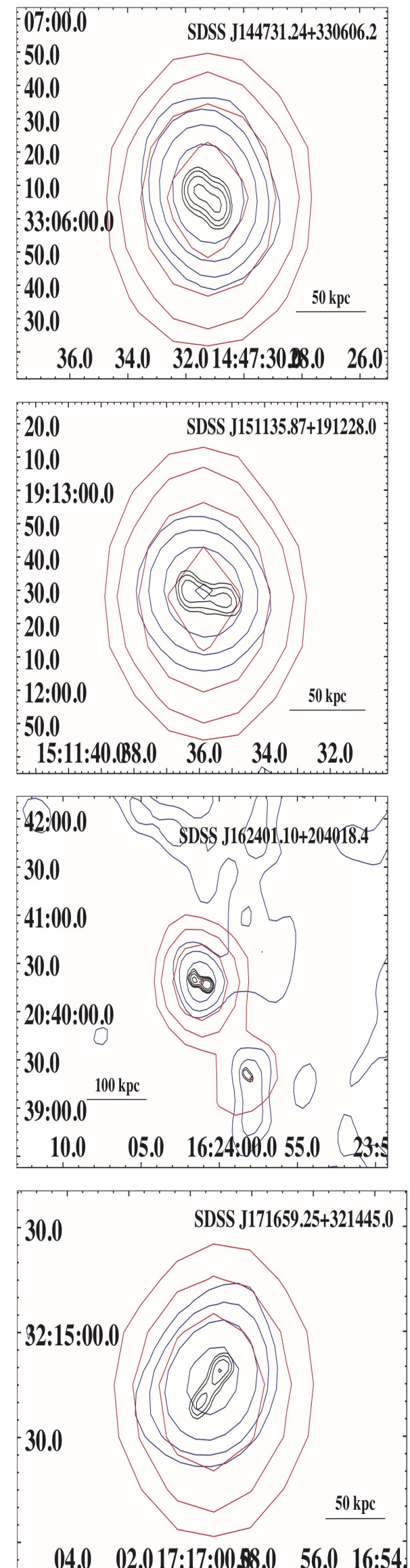

$\begin{array}{lllll}04.0 & 02.017: 17: 00 \$ 88.0 \quad 56.0 & 16: 54\end{array}$
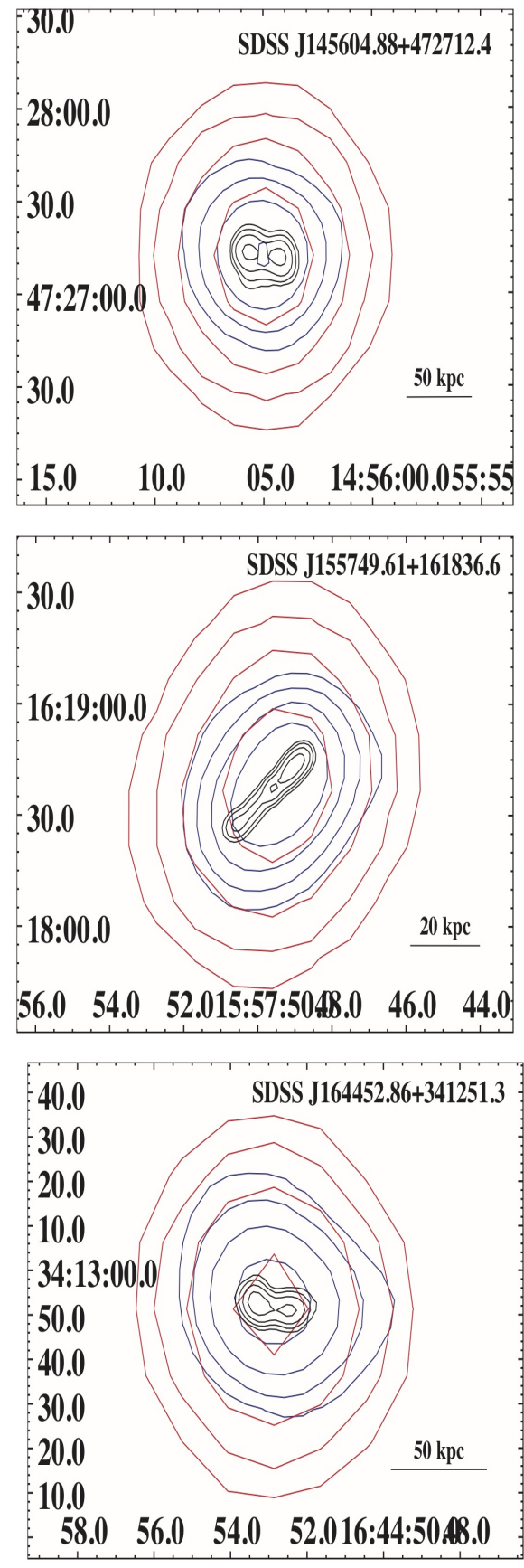
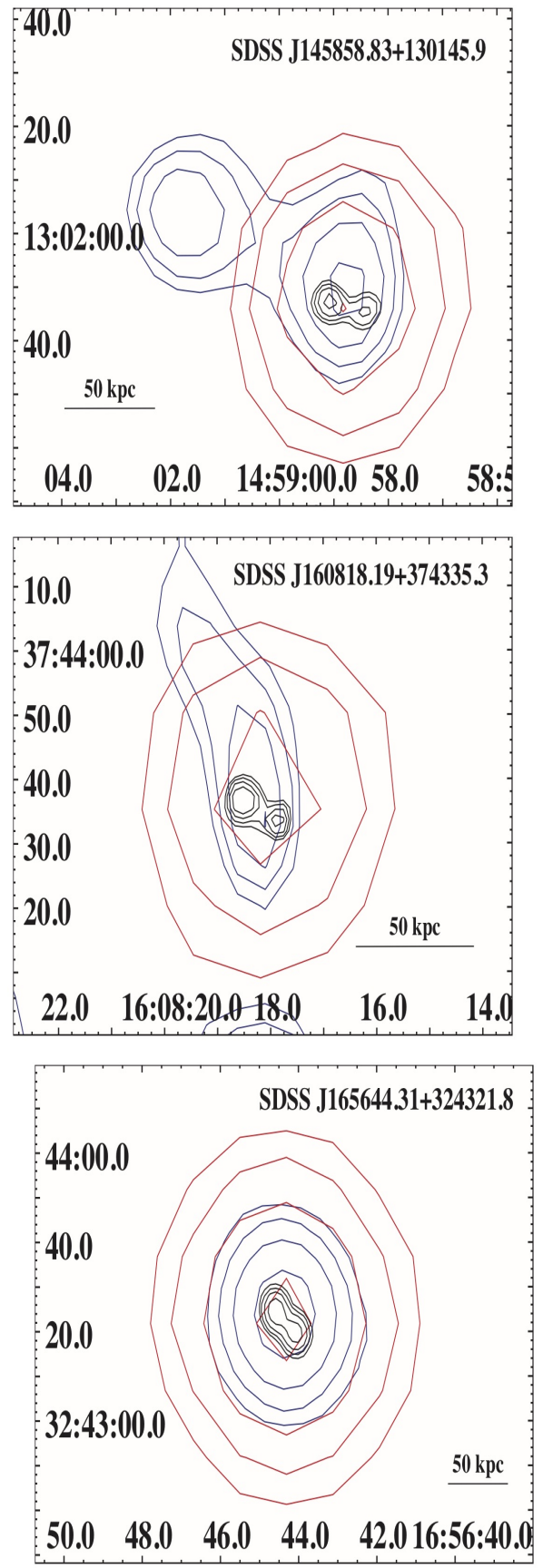

Fig. D.1. continued. 


\section{Appendix E: Images parameters: sources excluded and COMP2CAT sources}

Table E.1. Parameters of the images of the sources excluded because of their high-resolution radio morphologies.

\begin{tabular}{cccc}
\hline \hline \multirow{2}{*}{$\begin{array}{c}c \\
\text { name }\end{array}$} & $l$ FIRST & VLA \\
\cline { 2 - 3 } band & 1 & $f$ & band [GHz] \\
\hline $\mathrm{J} 111025.09+032138.8$ & 0.6 & 2 & 1.4 \\
$\mathrm{~J} 125724.35+272952.1$ & 1 & 3 & 1.4 \\
$\mathrm{~J} 125935.70+275733.3$ & 0.8 & 4 & 4.8 \\
$\mathrm{~J} 132451.44+362242.7$ & 2 & 2.25 & 1.4 \\
$\mathrm{~J} 161531.36+272657.3$ & & 4.8 \\
\hline
\end{tabular}

Notes. Column (1): SDSS name of the sources. Column (2): $l$, value of the starting contour level of the FIRST radio map and $f$, factor increase of the FIRST radio contours. Column (3): same parameters as in Col. (2) for the NVSS radio map. Column (4): same parameters as in Col. (2) for the TGSS radio map.

Table E.2. Parameters of the images of the sources with large-scale extended emission.

\begin{tabular}{|c|c|c|c|c|c|c|}
\hline \multirow{2}{*}{$\begin{array}{l}\text { SDSS } \\
\text { name }\end{array}$} & \multicolumn{2}{|c|}{ FIRST } & \multicolumn{2}{|l|}{ NVSS } & \multicolumn{2}{|c|}{ TGSS } \\
\hline & $l[\mathrm{mJy} /$ beam $]$ & $f$ & $l[\mathrm{mJy} / \mathrm{beam}]$ & $f$ & $l[\mathrm{mJy} / \mathrm{beam}]$ & $f$ \\
\hline J083224.82+184855.4 & 0.6 & 1.75 & 10 & 2 & 10 & 2 \\
\hline J083830.99+194820.4 & 0.6 & 1.5 & 3 & 1.5 & 10 & 2 \\
\hline J091443.12+073544.9 & 0.5 & 1.25 & 3 & 1.5 & 4 & 1.5 \\
\hline J115905.68+582035.5 & 0.8 & 1.25 & 1 & 2.5 & 8 & 2 \\
\hline $\mathrm{J} 132345.01+313356.7$ & 0.6 & 2 & 4 & 2 & 6 & 2 \\
\hline $\mathrm{J} 152804.95+054428.1$ & 1 & 2.25 & 2 & 2 & 10 & 2 \\
\hline J215305.08-071106.9 & 1 & 1.75 & 2 & 2 & 6 & 2 \\
\hline
\end{tabular}

Notes. Column (1): SDSS name of the sources. Column (2): $l$, value of the starting contour level of the FIRST radio map and $f$, factor increase of the FIRST radio contours. Column (3): same parameters as in Col. (2) for the NVSS radio map. Column (4): same parameters as in Col. (2) for the TGSS radio map. 
A. Jimenez-Gallardo et al.: COMP2CAT: hunting compact double radio sources

Table E.3. Parameters of the images of COMP2CAT sources.

\begin{tabular}{|c|c|c|c|c|c|c|}
\hline \multirow{2}{*}{$\begin{array}{l}\text { SDSS } \\
\text { name }\end{array}$} & \multicolumn{2}{|c|}{ FIRST } & \multicolumn{2}{|l|}{ NVSS } & \multicolumn{2}{|c|}{ TGSS } \\
\hline & $l[\mathrm{mJy} /$ beam $]$ & $f$ & $l[\mathrm{mJy} /$ beam $]$ & $f$ & $l[\mathrm{mJy} /$ beam $]$ & $f$ \\
\hline J073600.87+273926.0 & 0.8 & 1.5 & 3 & 1.5 & 4 & 2 \\
\hline J074132.98+475215.6 & 0.5 & 1.5 & 2 & 2 & 10 & 2 \\
\hline J074641.45+184405.4 & 1 & 1.5 & 5 & 1.5 & 10 & 2 \\
\hline J081023.27+421625.8 & 0.4 & 2 & 3 & 1.5 & 15 & 1.5 \\
\hline J081104.30+355908.3 & 2 & 1.75 & 6 & 2 & - & - \\
\hline J082033.79+395142.4 & 0.6 & 2 & 5 & 2 & 8 & 2 \\
\hline J083053.58+231035.7 & 0.6 & 1.5 & 4 & 1.5 & 10 & 1.5 \\
\hline J084517.83+303027.4 & 0.6 & 1.5 & 2 & 1.5 & - & - \\
\hline J090311.14+540351.6 & 2 & 2 & 4 & 2 & 10 & 2 \\
\hline J091134.75+125538.1 & 4 & 1.75 & 2.5 & 4 & 10 & 4 \\
\hline J095341.37+014202.3 & 0.5 & 1.5 & 3 & 1.5 & 20 & 1.5 \\
\hline $\mathrm{J} 100622.41+301332.9$ & 1 & 2 & 2 & 2 & 10 & 2 \\
\hline $\mathrm{J} 101653.82+002857.0$ & 0.4 & 2 & 2 & 2 & 6 & 2 \\
\hline $\mathrm{J} 101944.27-003817.8$ & 0.4 & 1.75 & 2 & 2 & 6 & 2 \\
\hline $\mathrm{J} 103801.77+414625.8$ & 0.8 & 2 & 6 & 1.5 & 10 & 2 \\
\hline $\mathrm{J} 103842.52+120315.6$ & 1 & 2 & 4 & 2 & 2 & 1.5 \\
\hline $\mathrm{J} 104254.02+282559.0$ & 1 & 1.75 & 6 & 2 & 10 & 2 \\
\hline $\mathrm{J} 111109.58+393552.0$ & 1 & 2.25 & 4 & 2 & 10 & 2 \\
\hline $\mathrm{J} 113305.52+592013.7$ & 1 & 1.5 & 3 & 2 & 8 & 2 \\
\hline $\mathrm{J} 113643.49+545446.8$ & 1 & 2 & 2 & 2 & 8 & 2 \\
\hline $\mathrm{J} 115050.98-031113.0$ & 1.5 & 2 & 4 & 2 & 20 & 2 \\
\hline $\mathrm{J} 122208.81+073329.6$ & 0.8 & 1.75 & 2 & 2 & 8 & 2 \\
\hline $\mathrm{J} 125319.21+475335.2$ & 0.6 & 2 & 4 & 1.5 & 8 & 1.5 \\
\hline J130107.54-4032652.5 & 1 & 3 & 4 & 3 & 8 & 3 \\
\hline $\mathrm{J} 131705.93+435713.2$ & 0.4 & 2 & 4 & 2 & 20 & 1.5 \\
\hline $\mathrm{J} 131945.31+603043.0$ & 0.6 & 4 & 4 & 3 & 8 & 2 \\
\hline $\mathrm{J} 132031.47-012718.5$ & 0.6 & 1.5 & 1 & 2 & 4.5 & 1.25 \\
\hline $\mathrm{J} 132602.39+364759.3$ & 1 & 4 & 10 & 4 & 10 & 4 \\
\hline $\mathrm{J} 132649.30+164948.0$ & 0.6 & 2 & 6 & 2 & 20 & 1.5 \\
\hline J133917.34-015048.7 & 1 & 2.5 & 4 & 2 & 20 & 2 \\
\hline $\mathrm{J} 135338.43+360802.4$ & 0.45 & 2 & 4 & 2 & 10 & 2 \\
\hline $\mathrm{J} 135347.34+515734.3$ & 1 & 2.75 & 4 & 2.5 & 10 & 4 \\
\hline $\mathrm{J} 144647.43+032527.1$ & 1 & 2.25 & 3 & 2 & 10 & 1.5 \\
\hline $\mathrm{J} 144731.24+330606.2$ & 1 & 2.5 & 6 & 2 & 10 & 2 \\
\hline $\mathrm{J} 145604.88+472712.4$ & 1 & 3 & 4 & 3 & 20 & 3 \\
\hline $\mathrm{J} 145858.83+130145.9$ & 1 & 1.5 & 3 & 1.5 & 10 & 1.25 \\
\hline $\mathrm{J} 151135.87+191228.0$ & 0.8 & 2 & 2 & 2 & 10 & 2 \\
\hline $\mathrm{J} 155749.61+161836.6$ & 3 & 1.75 & 4 & 2.5 & 10 & 2 \\
\hline $\mathrm{J} 160818.19+374335.3$ & 0.5 & 1.25 & 2 & 1.5 & 6 & 1.15 \\
\hline $\mathrm{J} 162401.10+204018.4$ & 0.8 & 1.5 & 2 & 2 & 4 & 2 \\
\hline $\mathrm{J} 164452.86+341251.3$ & 1 & 1.75 & 3 & 2 & 6 & 2 \\
\hline $\mathrm{J} 165644.31+324321.8$ & 2 & 1.75 & 5 & 2 & 10 & 2 \\
\hline $\mathrm{J} 171659.25+321445.0$ & 1 & 1.5 & 2 & 2 & 4 & 2 \\
\hline
\end{tabular}

Notes. Column (1): SDSS name of the sources. Column (2): $l$, value of the starting contour level of the FIRST radio map and $f$, factor increase of the FIRST radio contours. Column (3): same parameters as in Col. (2) for the NVSS radio map. Column (4): same parameters as in Col. (2) for the TGSS radio map. 